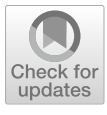

Cite as

Nano-Micro Lett.

(2020) $12: 151$

Received: 1 May 2020

Accepted: 18 June 2020

Published online: 15 July 2020

(C) The Author(s) 2020

\section{Self-Assembly Protein Superstructures as a Powerful Chemodynamic Therapy Nanoagent for Glioblastoma Treatment}

\author{
Tao Zheng ${ }^{1 凶}$, Wentao Wang ${ }^{1}$, Jon Ashley ${ }^{1}$, Ming Zhang ${ }^{1 凶}$, Xiaotong Feng ${ }^{1}$, \\ Jian Shen ${ }^{2,3}$, Yi Sun ${ }^{1} \bowtie$ \\ $\triangle$ Tao Zheng, taozhe@dtu.dk; Ming Zhang,mzhan@dtu.dk; Yi Sun, suyi@dtu.dk \\ 1 Department of Health Technology, Technical University of Denmark, 2800 Kongens Lyngby, Denmark \\ 2 Jiangsu Collaborative Innovation Center for Biomedical Functional Materials, School of Chemistry \\ and Materials Science, Nanjing Normal University, Nanjing 210023, People's Republic of China \\ 3 Key Laboratory of High Performance Polymer Material and Technology of Ministry of Education, School \\ of Chemistry and Chemical Engineering, Nanjing University, Nanjing 210023, People's Republic of China
}

\title{
HIGHLIGHTS
}

- A new type of protein nanostructure as a chemodynamic therapy nanoagent was fabricated via proper assembling and crosslinking techniques.

- The as-fabricated nanostructures could cross the blood-brain barrier, possessing excellent long circulating times and accumulation properties at the tumor site.

- The RBC@Hb@GOx NPs can be regarded as 'pure particles of drugs,'which can produce toxic reactive oxygen species to inhibit the growth of orthotopic brain tumor.

\begin{abstract}
Glioblastoma (GBM) remains a formidable challenge in oncology. Chemodynamic therapy (CDT) that triggers tumor cell death by reactive oxygen species (ROS) could open up a new door for GBM treatment. Herein, we report a novel CDT nanoagent. Hemoglobin $(\mathrm{Hb})$ and glucose oxidase (GOx) were employed as powerful CDT catalysts. Instead of encapsulating the proteins in drug delivery nanocarriers, we formulate multimeric superstructures as self-delivery entities by crosslinking techniques. Red blood cell (RBC) membranes are camouflaged on the protein superstructures to promote the delivery across blood-brain barrier. The as-prepared RBC@Hb@GOx nanoparticles (NPs) offer superior biocompatibility, simplified structure, and high accumulation at the tumor site. We successfully demonstrated that the NPs could efficiently produce toxic ROS to kill U87MG cancer cells in vitro and inhibit the growth of GBM tumor in vivo, suggesting that the new CDT nanoagent holds great promise for treating GBM.
\end{abstract}

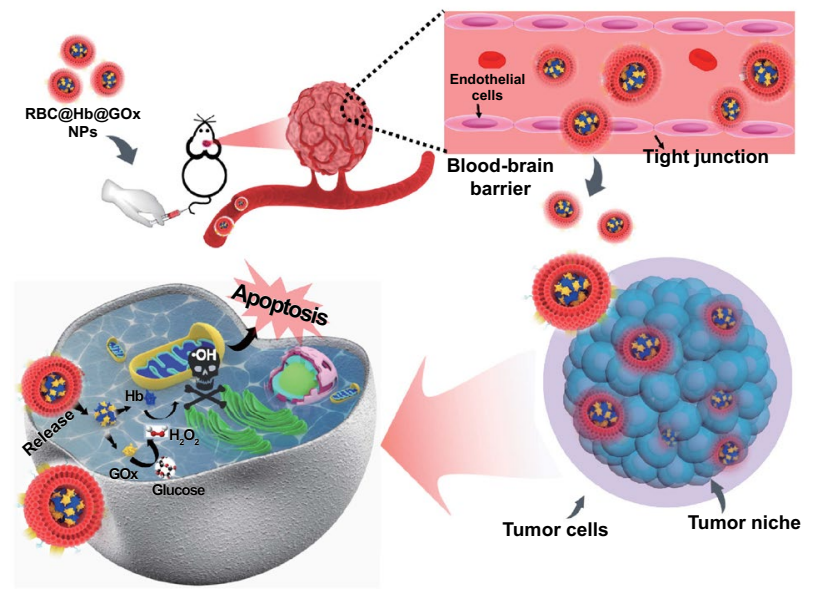

KEYWORDS Self-assembly protein superstructures; Glioblastoma therapy; Chemodynamic therapy; Self-delivery entities; Bloodbrain barrier 


\section{Introduction}

Glioblastoma multiforme (GBM) is the most malignant cancer in our central nervous system (CNS) [1]. It has an incidence of three per 100,000 adults per year and accounts for $52 \%$ of all primary brain tumors. The mainstay of treatment is surgery [2], followed by radiation and chemotherapy [3, 4]. Due to the aggressive nature and the localization in brain, standard therapies are ineffective in managing the devastating disease. Most GBM tumors are infiltrating and grow into the normal brain tissues, making it difficult to eliminate the cancerous tissues by surgery [5]. The efficacy of radiotherapy and chemotherapy is largely limited by the phenotypic and genotypic heterogeneity, hypoxic tumor microenvironment (TME), and intrinsic cell resistance mechanisms [6]. Translation of immunotherapy to GBM is also a distinct challenge, as the CNS is traditionally considered immune privileged [7]. With a 5-year survival rate of $<10 \%$ and median survival of only 15 months, GBM remains a formidable challenge in the field of oncology [8]. Developing new treatments for GBM will be of high importance for both the patients and the healthcare system.

Recently, chemodynamic therapy (CDT) has emerged as a new reactive oxygen species (ROS)-mediated therapeutic strategy. It employs Fenton-type reactions to convert intracellular hydrogen peroxide $\left(\mathrm{H}_{2} \mathrm{O}_{2}\right)$ into highly cytotoxic hydroxyl radial $(\cdot \mathrm{OH})$, triggering the death of cancer cells through the apoptosis or necrosis processes [9-13]. With the ability to modulate ROS in TME, CDT might open up new opportunities for GBM treatment. Nevertheless, despite the fact that various sophisticated CDT nanoagents have been engineered, CDT is still in its infancy and has seldom been used in brain cancers. A number of critical issues including toxicity of the nanomaterials, complexity of the structures, and difficulty in penetrating BBB must be addressed before applying CDT in clinical practice.

To realize CDT, it is important to select materials with excellent Fenton catalytic activity $[14,15]$. Up to now, mainly metal-based inorganic materials and metal-organic framework (MOF) have been exploited as catalysts [16, 17]. Unfortunately, safety remains the predominant concern for the CDT nanoagents. It is known that metallic nanoparticles are prone to elicit toxic responses in the brain. Shao et al. concluded that the autophagy, inflammatory response, and disturbed signaling pathways might be the main mechanisms underlying the neurotoxicity of metallic nanoparticles [18]. Moreover, at physiological conditions, most organic/inorganic Fenton catalysts are non-biodegradable. They may persist in the body for long periods, causing systematic toxicity due to prolonged exposure to different biological microenvironments. Thus, it is imperative to create highly biocompatible and biodegradable Fenton catalysts to minimize nerotoxicity and systemic toxicity.

Besides the toxicity, another challenge of the CDT nanoagent is their complex structures. Typical CDT nanoagents consist of three major components: Fenton catalysts, nanocarriers, and modifiers/targeting ligands coated on outer surface. Several nanocarriers made of a zeolitic imidazolate framework [19, 20], block copolymers [21-23], or dendritic mesoporous silica [24, 25] have been devised to encapsulate Fenton catalysts. These fancy materials and structures demonstrated the advances in nanotechnology. However, the complicated formulations, tedious synthesis processes, and instability of the colloids have become the major hurdles in the clinical translation of CDT. Therefore, useful CDT nanoagents must possess characteristics of ease of synthesis, simplified structures, and enhanced stability.

In addition to the drawbacks of the CDT nanoagents, developing CDT for GBM treatment is further hampered by the presence of the highly restrictive blood-brain tumor barrier and blood-brain tumor barrier (BBTB) $[26,27]$. The $\mathrm{BBB} / \mathrm{BBTB}$ can limit the penetration of therapeutics from the systemic circulation into the tumor compartment [28]. One popular approach to facilitate the drug delivery across $\mathrm{BBB} / \mathrm{BTB}$ is receptor-mediated transcytosis, which hijacks the receptors/transporters expressed on the barriers [29, 30]. Different ligands have been reported to actively target the transferrin receptors, insulin-like growth factors [31], or low-density lipoprotein receptors [32]. However, these receptors are ubiquitously expressed in several cell types and tissues, leading to unwanted peripheral organ uptake. Moreover, the transport capacity of endocytosis is rather low due to the limited number of membrane transport proteins and the complex intracellular trafficking procedures. The best ligand published so far only delivered less than $1 \%$ of the injected dose to brain, with even less ending up at the tumor site [33]. As such, increasing the accumulation of Fenton agents at GBM would greatly improve the outcome of CDT. 
In this study, to tackle the major obstacles in CDT, we constructed a new type of CDT nanoagent (Fig. 1a). The potential toxicity of inorganic nanomaterials and MOFs have urged us to turn our attention to the plethora of natural proteins. In fact, a large number of proteins in nature, the so-called metalloproteins, contain metal ion cofactors, which can act as Fenton catalytic ions, such as $\mathrm{Fe}^{2+}, \mathrm{Cu}^{2+}, \mathrm{Mn}^{2+}$, and $\mathrm{Co}^{2+}$. Examples include $\mathrm{Hb}$ [34] nitrite reductase [35] and ceruloplasmin [36]. In addition, some proteins are able to generate exogenous $\mathrm{H}_{2} \mathrm{O}_{2}$, such as $\mathrm{GOx}$ [37] and NADPH oxidase [38]. In this study, we showed that the combination of $\mathrm{Hb}$ and GOx could be an attractive alternative to the synthetic Fenton catalysts. The predominant advantages offered by the natural proteins are the high biocompatibility and biodegradability. Employing them for CDT significantly improves the safety of the therapy.

To co-deliver the two proteins-Hb and GOx, instead of loading them into the nanocarriers, we proposed a new concept of 'self-delivery.' With proper assembling and crosslinking techniques, the two protein monomers were formulated into multimeric protein superstructures (denoted as $\mathrm{Hb} @ \mathrm{GOx} \mathrm{NPs}$ ), thus eliminating the need of the nanocarrier component in conventional drug delivery systems. The protein superstructures present a unique way to deliver multiple proteins. They can be regarded as 'pure particles of drugs,' which greatly simplifies the structure and the synthesis steps and offers extremely high drug loading yields. In addition, the stability of the protein nanoparticles is much more enhanced compared to free proteins loosely encapsulated in the carriers.

To facilitate the delivery of Hb@GOx NPs to the GBM microenvironment, we employed a passive targeting strategy to cross $\mathrm{BBB} / \mathrm{BBTB}$. It has been shown that for disorders such as GBM, the integrity of the BBB is partially compromised. Hence, passive targeting is possible if the size of NPs is sufficiently small and the circulation time is long. We camouflaged the protein superstructures with red blood cell (RBC) membranes (denoted as RBC@Hb@GOx NPs). The CD 47 protein and glycosyl groups on the membrane surface endow the nanoparticles with prolonged systematic retention time, less reticuloendothelial system uptake, and reduced immuno-recognition [39, 40]. Compared to the active targeting, passive targeting would lead to enhanced delivery efficiency.
The working mechanism of the novel CDT nanoagent is shown in Fig. 1b. The RBC@Hb@GOx NPs are administrated intravenously and circulate with the blood. The RBC membrane helps with passive diffusion across the endothelial cells of BBB/BBTB. Once reaching the tumor site, the nanoagents are uptaken by the tumor cells. The abundant in situ $\mathrm{H}_{2} \mathrm{O}_{2}$ causes cell membrane destruction, which increases the access of $\mathrm{Hb} @$ GOx protein superstructures. The two electron oxidation of $\mathrm{Fe}(\mathrm{II})-\mathrm{Hb}$ by $\mathrm{H}_{2} \mathrm{O}_{2}$ produces the $\mathrm{Fe}(\mathrm{IV})$-ferrylHb that can react with an additional molecule of $\mathrm{H}_{2} \mathrm{O}_{2}$, resulting in the degradation of heme groups and the release of free ions. The ions catalyze Fenton reactions, converting $\mathrm{H}_{2} \mathrm{O}_{2}$ to toxic $\cdot \mathrm{OH}$ and $\mathrm{O}_{2}^{--}$(Fig. $1 \mathrm{~b}$, Eqs. 1-6). In the meantime, GOx consumes oxygen and glucose to generate $\mathrm{H}_{2} \mathrm{O}_{2}$ and gluconic acid. The increased acidity and $\mathrm{H}_{2} \mathrm{O}_{2}$ in the microenvironment further boost the Fenton reactions. Eventually, the protein superstructures are degraded, and the tumor cells are killed by the excessive oxidative stress. In this paper, we successfully proved that the novel RBC@Hb@GOx NPs could inhibit the growth of GBM in mice models. To our best knowledge, this is the first time that the CDT nanoagent was constructed with $100 \%$ natural biomaterials and the in vivo therapeutic efficacy of CDT was demonstrated in GBM.

\section{Experimental Section}

\subsection{Preparation of Hb@GOx NPs}

$\mathrm{Hb}$ powder $(25 \mathrm{mg})$ and $\mathrm{GOx}(15 \mathrm{mg})$ powder were added to MQ water $(6 \mathrm{~mL})$ and stirred overnight at $500 \mathrm{rpm}$ using a magnetic stirrer for complete hydration. Afterward, $10 \mu \mathrm{L}$ of $2 \%$ glutaraldehyde solution was mixed to induce intraparticle crosslinking. The solution was stirred continuously at $500 \mathrm{rpm}$ at room temperature for $24 \mathrm{~h}$.

\subsection{Preparation of ICG-Loaded Hb@GOx NPs}

$\mathrm{Hb}$ powder $(25 \mathrm{mg}), \mathrm{GOx}(15 \mathrm{mg})$, and $6 \mathrm{mg}$ ICG powder $(6 \mathrm{mg})$ were added to MQ water $(6 \mathrm{~mL})$ and then were stirred overnight at $500 \mathrm{rpm}$ using a magnetic stirrer for complete hydration. The following process was the same as mentioned above. 
(a)<smiles>O=CCCCCC=O</smiles>

Natural red blood cells Red blood cells membrane (RBC)

RBC@Hb@GOx NPs
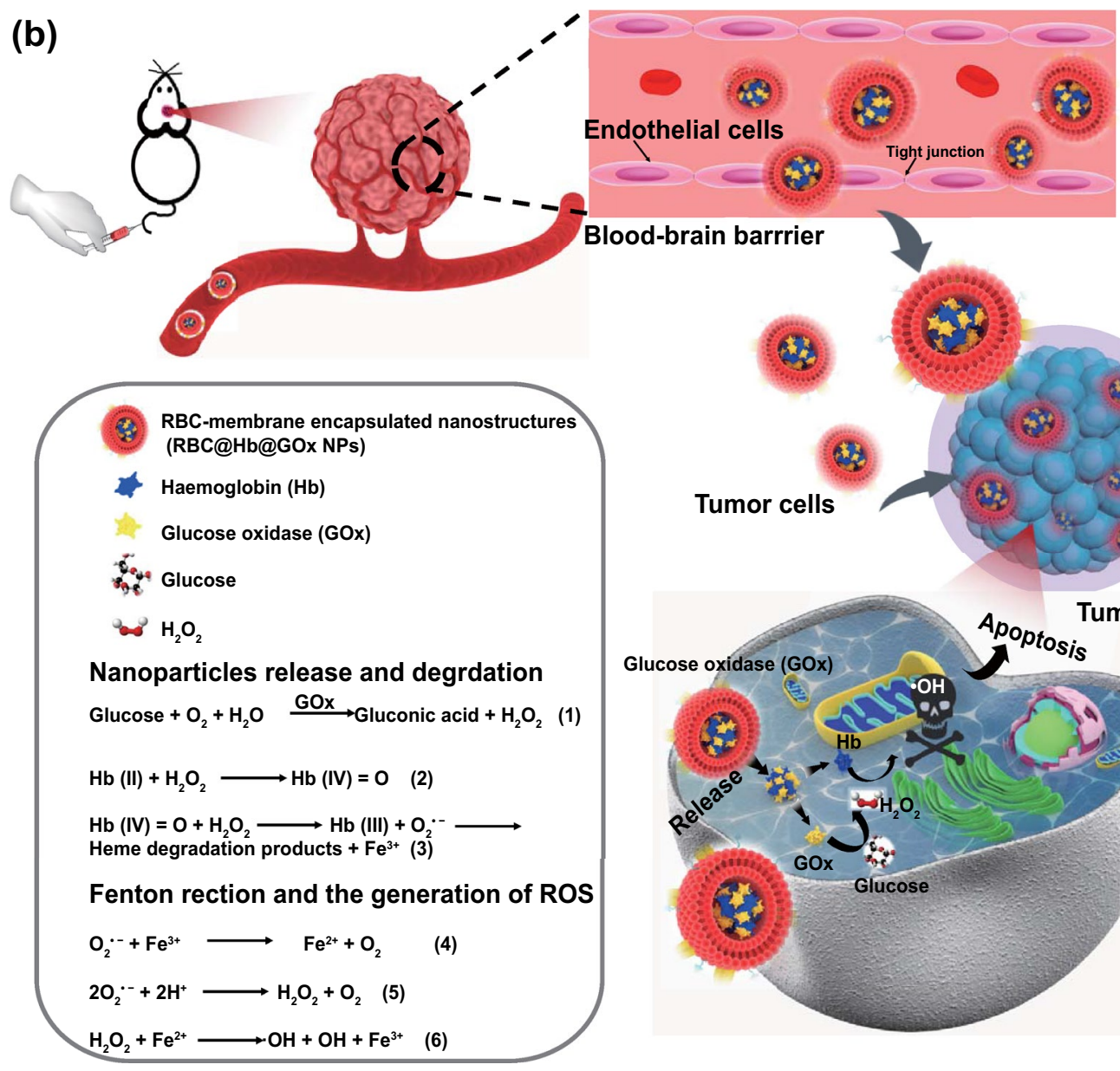

Blood-brain barrrier

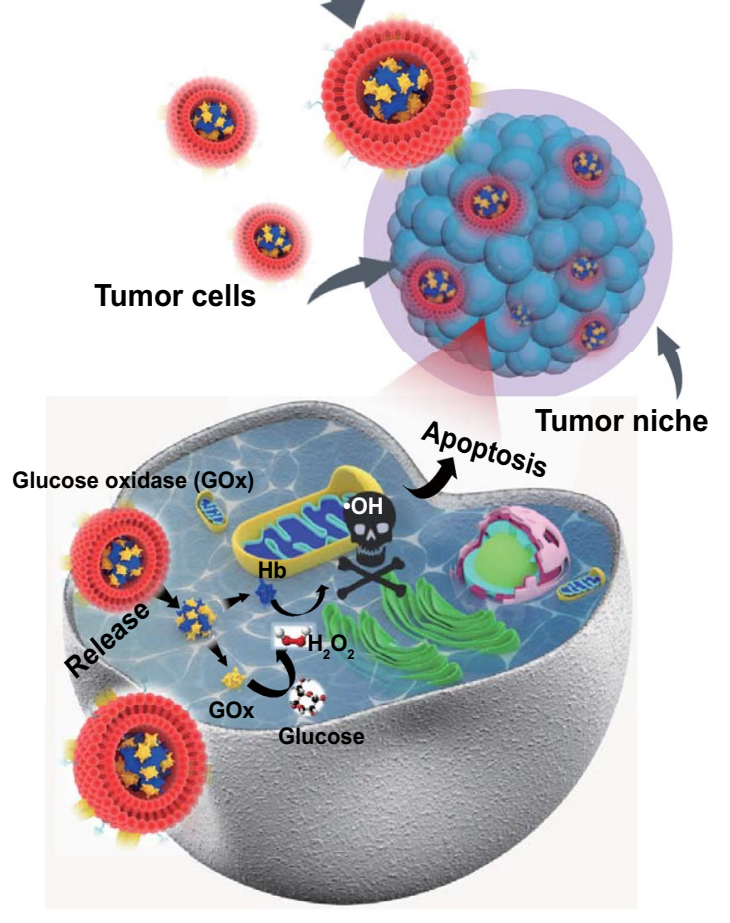

Fig. 1 Design of hemoglobin, gluocose oxidase-based protein superstructures as a powerful chemodynamic therapy nanoagent for glioblastoma treatment. a Schematic illustration of synthesizing RBC@Hb@GOx NPs. b Scheme of TME-activated ROS production by RBC@Hb@GOx NPs for GBM treatment 


\subsection{Preparation of RBCs Membrane}

Briefly, the blood was taken from the cattle and diluted by PBS (pH 7.4). After centrifuging for $5 \min \left(3000 \mathrm{rpm}, 4^{\circ} \mathrm{C}\right)$, the collected RBCs were washed 3 times (pH 7.4), re-dispersed in the water and placed in the ice bath for $50 \mathrm{~min}$. Finally, the solution was centrifuged $\left(12,000 \mathrm{rpm}, 4{ }^{\circ} \mathrm{C}\right)$ and then washed with PBS ( $\mathrm{pH}$ 7.4) until the supernatant became colorless. The obtained RBCs membrane was re-dispersed in PBS (pH 7.4), sonicated, and filtered through a $0.45 \mu \mathrm{m}$ and $0.22 \mu \mathrm{m}$ filter before use.

\subsection{Preparation of RBC Membrane Modifying Hb@ GOx or ICG-Loaded Nanoparticles}

$100 \mu \mathrm{L}$ of RBC membrane was added to $400 \mu \mathrm{L}$ of MQ water. The solution was sonicated for $10 \mathrm{~s}$ before being mixed with $500 \mu \mathrm{L}$ of Hb@GOx NPs or ICG-loaded Hb@ GOx NPs. The mixture solution was sonicated for $60 \mathrm{~s}$ to complete the membrane coating. The excess RBC membrane was removed by centrifugation, and RBCs@Hb@GOx NPs or ICG-RBCs@Hb@GOx NPs were re-dispersed in MQ water for further use.

\subsection{Evaluation the Generation of $\mathrm{H}_{2} \mathrm{O}_{2}$ In Vitro}

The catalytic performance of RBCs@Hb@GOx NPs in the presence of glucose $(10 \mathrm{mM})$ was conducted using fluorimetric hydrogen peroxide assay kit. The produced $\mathrm{H}_{2} \mathrm{O}_{2}$ generates a red fluorescent product $\left(\lambda_{\mathrm{ex}}=540 / \lambda_{\mathrm{em}}=590 \mathrm{~nm}\right)$. At each time point $(0,10,20,30$, and $45 \mathrm{~min})$, the production of $\mathrm{H}_{2} \mathrm{O}_{2}$ was calculated according to the fluorescence standard curve obtained from a fluorescent microplate reader. In addition, the UV-Vis absorbance of the mixed solution at $425 \mathrm{~nm}$ was measured to further investigate the degradation of RBCs@Hb@GOx NPs at room temperature.

\subsection{Detection of Hydroxyl Radicals In Vitro}

The 1,3-diphenylisobenzonfuran (DPBF, Sigma-Aldrich), as a hydroxyl radical indicator, was applied to investigate the generation of hydroxyl radicals via measuring the absorption intensity at wavelength of $410 \mathrm{~nm}$ in UV-Vis absorption spectrum. Firstly, the test solution (RBCs@Hb@GOx NPs mixed with glucose) was prepared, and samples were collected at reaction time points of $0,10,15,20$, and $25 \mathrm{~min}$. After that, DPBF $(100 \mu \mathrm{L})$ in methanol $(20 \mathrm{mM})$ was added into the sample solutions $(100 \mu \mathrm{L})$. The absorption intensity of DPBF was recorded. At same time, the group of DPBF solution treated with $\mathrm{Hb}$ plus $\mathrm{H}_{2} \mathrm{O}_{2}$ solution was set as the control group.

\subsection{Electron Spin Resonance (ESR) Measurements for the Detection of Hydroxyl Radicals $(\cdot \mathbf{O H})$ and Superoxide Radical $\left(\mathrm{O}_{2}^{-}\right)$In Vitro}

For detecting $\cdot \mathrm{OH}$ detection, BMPO $\left(25 \mathrm{mM}, 50 \mu \mathrm{g} \mathrm{mL}^{-1}\right)$ in PBS, Hb@GOx NPs, and RBCs@Hb@GOx NPs mixed with glucose $(10 \mathrm{mM})$ or without glucose (as control group) were prepared. ESR spectra were recorded after incubation of 6 min. For detecting $\mathrm{O}_{2}^{--}$, BMPO $\left(25 \mathrm{mM}, 50 \mu \mathrm{g} \mathrm{m}^{-1}\right)$ in PBS, Hb@GOx NPs and RBCs@Hb@GOx NPs mixed with glucose (10 mM) or without glucose (as control group) were prepared. ESR spectra were recorded after incubation of $6 \mathrm{~min}$.

\subsection{Cell Culture}

NIH3T3 and U87MG cell lines were obtained from the American Type Culture Collection (ATCC). All cell lines were stored in a $100 \%$ humidity atmosphere of $37{ }^{\circ} \mathrm{C}$ and $5 \% \mathrm{CO}_{2}$.

\subsection{In Vitro Cytotoxicity Assay}

MTT assay was applied to evaluate the cytotoxicity of RBCs@Hb@GOx NPs. Firstly, NIH3T3 or U87MG cells were randomly seeded into 96 -well plates and incubated with RBCs@Hb@GOx NPs at different concentrations $(0,50,100,200,300,400,500,600,700,800$, and $1000 \mu \mathrm{g} \mathrm{mL}^{-1}$ ) at $100 \%$ humidity atmosphere at $37^{\circ} \mathrm{C}$ and $5 \% \mathrm{CO}_{2}$ for $24 \mathrm{~h}$. Then, $20 \mu \mathrm{L}$ of MTT solution was added into each well, placed in $\mathrm{CO}_{2}$ incubator for further incubation for $4 \mathrm{~h}$. DMSO $(150 \mu \mathrm{L})$ was added, and the absorbance at $490 \mathrm{~nm}$ was obtained to calculate cell survival rate by using MTT assay kit. 


\subsection{Hemolysis Assay}

Firstly, the obtained cattle blood red cells were washed with PBS by centrifuging. Next, the properly diluted blood samples were added into the RBCs@Hb@GOx NPs solution with different sample concentrations $\left(500-2500 \mu \mathrm{g} \mathrm{mL}{ }^{-1}\right)$. After being stationary in PBS (negative controls) and deionized water (positive controls) for $1 \mathrm{~h}$, the supernatant was obtained after centrifuging and was further accessed to calculate the hemolysis ratio.

\subsection{In Vitro Chemodynamic Therapy for U87MG Cells}

U87MG cells $\left(1 \times 10^{4}\right.$ cells per well $)$ were seeded into a 96-well and incubated with PBS, Hb, GOx, Hb@GOx NPs, RBCs@Hb@GOx NPs, $\mathrm{Hb}$ and $\mathrm{H}_{2} \mathrm{O}_{2}, \mathrm{GOx}$ and $\mathrm{H}_{2} \mathrm{O}_{2}, \mathrm{Hb} @$ GOx NPs and $\mathrm{H}_{2} \mathrm{O}_{2}$, RBC@ $\mathrm{Hb} @ G O x$ NPs and $\mathrm{H}_{2} \mathrm{O}_{2}, \mathrm{Hb}$ and glucose, GOx and glucose, Hb@GOx NPs and glucose, RBC@Hb@GOx NPs and glucose at 100\% humidity atmosphere of $37{ }^{\circ} \mathrm{C}$ and $5 \% \mathrm{CO}_{2}$ for $24 \mathrm{~h}$, respectively. Next, 20 $\mu \mathrm{L}$ of MTT solution was added into each well and placed in $\mathrm{CO}_{2}$ incubator for further incubating for $4 \mathrm{~h}$. DMSO (150 $\mu \mathrm{L}$ ) was added, and the absorbance at $490 \mathrm{~nm}$ was obtained to calculate cell survival rate using the MTT assay kit. Moreover, in order to investigate the chemodynamic efficacy of RBC@Hb@GOx NPs on U87MG cells in vitro, CalceinAM $(100 \mu \mathrm{L})$ and PI solution $(100 \mu \mathrm{L})$ were incubated with U87MG cells for 30 min. Living cells were stained with calcein-AM (green fluorescence) and dead cells with PI (red fluorescence)

\subsection{Evaluation of the Production of Intracellular ROS}

The U87MG cells were randomly seeded into 6-well plates and then incubated with different groups under DMEM at a $100 \%$ humidity atmosphere at $37^{\circ} \mathrm{C}$ and $5 \% \mathrm{CO}_{2}$ for $24 \mathrm{~h}$. After that, the cells were washed with PBS (centrifuged at $1000 \mathrm{rpm}$ for $5 \mathrm{~min}$ ), adjusting the cell concentration to $1 \times 10^{6} \mathrm{~mL}^{-1}$. DCFH-DA was diluted with serum-free culture solution at 1:1000 to achieve a final concentration of $10 \mu \mathrm{M}$. Then, the collected cells were suspended in diluted DCFH-DA solution and further incubated at $37{ }^{\circ} \mathrm{C}$ for $20 \mathrm{~min}$. Besides, the incubation solution was shaken to make the probe fully contact with the cell. Finally, the cells were washed with serum-free cell culture medium 3 times to fully remove DCFH-DA that did not enter into cells. The fluorescence intensity of cells was determined by confocal fluorescence microscope.

Quantitative analyses for ROS production were analyzed by flow cytometric (Becton-Dickinson FACS Calibur) experiments. The U87MG cells after being treated with different groups were incubated with $10 \mu \mathrm{M}$ of DCFH-DA for $20 \mathrm{~min}$. Then, the incubation solutions were analyzed by flow cytometry with excitation at $488 \mathrm{~nm}$ and emission at $530 \mathrm{~nm}$.

\subsection{Evaluation of Mitochondrial Damage In Vitro}

The U87MG cells were randomly seeded into 6-well plates and then incubated with different groups under 90\% DMEM plus $10 \% \mathrm{FBS}$ at a $100 \%$ humidity atmosphere of $37{ }^{\circ} \mathrm{C}$ and $5 \% \mathrm{CO}_{2}$ for $24 \mathrm{~h}$. The logarithmic growth cells were seeded into six-well plate. When the cells grew adhering to the wall after 24 incubation, the corresponding drug-containing medium was added according to the group setting, and a negative control group was set up. Then, the cells were washed with PBS (centrifuged at $2000 \mathrm{rpm}$ for $5 \mathrm{~min}$ ), adjusting the cell concentration to $1 \times 10^{6} \mathrm{~mL}^{-1}$. Next, the $100 \mu \mathrm{L}$ of $10 \times$ buffer solution and $900 \mu \mathrm{L}$ of sterilized deionized water were diluted into $1 \times$ Buffer, mixed and preheated to $37^{\circ} \mathrm{C}$. JC-1 working solution was formed via absorbing $500 \mu \mathrm{L}$ of incubation buffer $(1 \times$ Buffer $)$ into $1 \mu \mathrm{L}$ of JC-1 solution. After that, $500 \mu \mathrm{L}$ of JC-1 working solution was suspended with cells and incubated them at $37{ }^{\circ} \mathrm{C}$ and 5\% $\mathrm{CO}_{2}$ incubator for 15-20 min. Finally, cells treated with different groups were collected by centrifugation at room temperature (1000 rpm, $5 \mathrm{~min}$ ) and washed twice in $1 \times$ Buffer, and further analyzed by confocal fluorescence microscope and flow cytometry.

\subsection{HMGB1 Release Assay}

U87MG cells were seeded into in 6-well plates. After 24-h treatment with different groups, dead cells were removed by centrifugation and the supernatants were concentrated to $100 \mu \mathrm{L}$. Released HMGB1 in supernatants were tested via western blot. 


\subsection{Preparing Mouse Model}

U87MG human glioma cells were purchased from American Type Culture Collection (ATCC) and maintained in DMEM media (Gibco) with 10\% FBS (Hyclone). Six-week-old NCR nude female mice were used to generate intracranial orthotopic U87MG gliomas. Briefly, mice were anesthetized using $2 \%$ isoflurane and their heads were immobilized in a stereotactic headframe using atraumatic ear bars. A burr hole was made using a steel drill bit (Plastics One, Roanoke, VA, USA) $1.4 \mathrm{~mm}$ right of the sagittal and $1 \mathrm{~mm}$ anterior to the lambdoid suture. Tumors were allowed to grow for 14 days prior to treatment. Intracranial tumor growth was monitored in vivo using bioluminescence IVIS ${ }^{\circledR}$ imaging (Xenogen, Almeda, CA) equipped with LivingImage ${ }^{\mathrm{TM}}$ software (Xenogen).

\subsection{Evaluation of Transportability across the BBB In Vivo}

ICG-loaded Hb@GOx NPs or RBC@Hb@GOx NPs were employed to investigate the transportability across the BBB based on the ICG fluorescence at tumor site. The orthotopic U87MG gliomas (without self-fluorescence) bearing mice were intravenously injected with ICG-Hb@GOx NPs and ICG-RBC@Hb@GOx NPs $(250 \mu \mathrm{L})$. The fluorescence signal at brain was detected in vivo using bioluminescence IVIS $^{\circledR}$ imaging equipped with LivingImage ${ }^{\mathrm{TM}}$ software (Xenogen) at $0,6,12,36,48$, and $72 \mathrm{~h}$ post-injection. In addition, the main organs from the treated mice, including heart, liver, spleen, lung, kidney, and brain, were collected after $72 \mathrm{~h}$ injection for imaging and biodistribution analysis.

\subsection{Evaluation of Anti-tumor Efficiency In Vivo}

The orthotopic U87MG gliomas (with self-fluorescence) bearing mice were randomly divided into four groups: (1) PBS (as control group, $200 \mathrm{~mL})$; (2) GOx $(250 \mu \mathrm{L}$, $\left.40 \mathrm{mg} \mathrm{kg}^{-1}\right)$; (3) Hb@GOx NPs $\left(250 \mu \mathrm{L}, 40 \mathrm{mg} \mathrm{kg}^{-1}\right)$; and (4) RBC@Hb@GOx NPs $\left(250 \mu \mathrm{L}, 40 \mathrm{mg} \mathrm{kg}^{-1}\right)$. Treatment began from day 21 after tumor implantation, and all treatments were intravenously administered. They were administered once every 10 days. After treatment with above groups, tumor response to treatment was tracked using IVIS imaging. Signal intensity was quantified within a region of interest using Living Image ${ }^{\mathrm{TM}}$ software.

\subsection{In Vivo Biocompatibility Evaluation}

The slice from major organs and tumor was obtained from each group after 40 days. H\&E staining was conducted for histological examination and observed via Olympus BX43 microscope (Japan).

\subsection{TUNEL, H\&E and Congo Red Staining}

TUNEL Apoptosis Assay was used for determining tumor cells apoptosis. The orthotopic U87MG gliomas bearing mice brain tissue were stained with TUNEL Apoptosis Assay Kit after treatment. For histology, the frozen tissue sections of $4 \sim 5 \mu \mathrm{m}$ were immersed in $1 \%$ acetone fixation solution at room temperature for $5 \mathrm{~min}$. Afterwards the tissue sections were dried, washed with PBS (3 times) for $3 \mathrm{~min}$, and further stained with H\&E and Congo Red. Finally, the collected sections were analyzed by an Olympus BX43 microscope (Japan). All staining experiments were conducted following the standard protocol.

\subsection{Statistical Analysis}

The statistical analyses were conducted by Student's $t$ test. Differences were considered statistically significant at $p<0.05$.

\subsection{Ethical Approval}

All experiments involving animals were performed in compliance with relevant ethical regulations in adherence with the Nanjing University and Nanjing Normal University for the Care and Use of Laboratory Animals, Nanjing, China.

\section{Results and Discussion}

\subsection{Characterization of Hb@GOx NPs and RBC@ Hb@GOx NPs}

The key technical challenge here is to develop an appropriate method to construct the protein superstructures, so that their biological activities can be retained. There have 
been crosslinked protein nanoparticles derived from gelatin [41], albumin [42], and gliadin [43]. The existing fabrication methods such as coacervation and desolvation usually unfold the proteins to decrease the intramolecular hydrophobic interactions. They are not applicable as the functionality of the proteins is destroyed during the process. In this work, we developed a facile 'dissolving-crosslinking' approach to prepare the protein particulates under mild conditions without the use of harsh chemicals or organic solvents. The process for synthesizing Hb@GOx NPs and RBC@ $\mathrm{Hb} @$ GOx NPs is shown in Figs. 1a and S1. Briefly, Hb and GOx powder were added into MQ water, and then, the mixed solution was stirred overnight for complete hydration. Afterward, glutaraldehyde (GA) was added as crosslinker to form intermolecular interactions and induce the assembly of $\mathrm{Hb}$ and GOx. The low-degree crosslinking caused little loss to the bioactivities of the proteins. To investigate the whole assembly process and determine the size of as-fabricated nanoparticles, the dynamic light scattering (DLS) and transmission electron microscopy (TEM) were used to monitor the reaction at four time points $(3,6,12$, and $24 \mathrm{~h})$. With the increase in the reaction time, the NPs became bigger and more homogenous, as shown in Fig. 2a. We chose $24 \mathrm{~h}$ as the crosslinking time due to the uniform morphology observed via TEM. The representative TEM shows that the as-fabricated Hb@GOx NPs possess an average size of $34.03 \mathrm{~nm}$ (Fig. 2b, c) with good polydispersity index (PDI) of 0.435 (Fig. S2a). After camouflaged by the RBC membrane, the average size increased to $51.55 \mathrm{~nm}$ (Figs. 2d and S2b).

The Fourier transform infrared (FT-IR) spectrum is shown in Fig. 2e. The shapes of the infrared absorption bands of amide I and amide II in $\mathrm{Hb}$ molecule provided detailed information on the secondary structure of the polypeptide chain. The absorption band between 1700 and $1600 \mathrm{~cm}^{-1}$ belonging to amide $\mathrm{I}$ was attributed to $\mathrm{C}=\mathrm{O}$ stretching vibration of peptide linkages in the protein's backbone. The absorption band of $1620-1500 \mathrm{~cm}^{-1}$ for amide II was due to a combination of $\mathrm{N}-\mathrm{H}$ bending and $\mathrm{C}-\mathrm{N}$ stretching. Normally, the absorption bands of amide I and amide II are eliminated when the structure of $\mathrm{Hb}$ is denatured. Figure 2e displays the FT-IR spectra of Hb, GOx, and RBC@Hb@GOx NPs. The absorption bands for amide I and amide II in the RBC@ $\mathrm{Hb} @$ GOx NPs were located at 1643.91 and $1536.78 \mathrm{~cm}^{-1}$, respectively, which were nearly the same as those obtained for the native protein (1650.01 and $1536.79 \mathrm{~cm}^{-1}$ ) [44] The absorption bands of the RBC@Hb@GOx NPs in amide I drifted slightly from reference values, indicating an interaction between $\mathrm{Hb}$ and GOx. In addition, the absorption peaks at around 450 nm of RBC@Hb@GOx NPs in UV-Vis absorption spectrum was the same as Hb (Fig. 2f), suggesting that the structure of $\mathrm{Hb}$ was well maintained.

The X-ray photoelectron spectroscopy (XPS) was applied to analyze the existing elements in the as-prepared NPs. No obvious Fe peaks were detected from the samples without adding glucose solution (Fig. S3), indicating the tight combination between the $\mathrm{Fe}^{2+}$ and heme. After incubation with glucose solution, Fenton reactions occurred. The peaks belonging to $\mathrm{Fe}$ $2 p$ and $\mathrm{Fe} 3 p$ were detected (Fig. $2 \mathrm{~g}$ ), indicating that the $\mathrm{Fe}^{2+}$ was released from heme group (Fig. S4). Besides, the presence of peaks of $\mathrm{Fe} 2 p, \mathrm{Fe} 3 p, \mathrm{Fe} 2 s$, and $\mathrm{Fe} 3 s$ in the high-resolution XPS further verified the existence of $\mathrm{Fe}^{2+}$ and $\mathrm{Fe}^{3+}$ in the Fenton reaction (Fig. S5). The zeta potential was measured after each synthesis procedure, with a value of $4.79 \pm 1.05 \mathrm{mV}$ for $\mathrm{Hb},-5.70 \pm 2.09 \mathrm{mV}$ for $\mathrm{GOx},-4.59 \pm 0.1 \mathrm{mV}$ for $\mathrm{Hb} @$ $\mathrm{GOx},-7.22 \pm 0.75 \mathrm{mV}$ for RBCs, and $-14.867 \pm 0.33 \mathrm{mV}$ for RBC@Hb@GOx NPs (Figs. 2h and S6). Finally, western blotting (WB) assay was applied to further confirm the existence of the CD47 membrane proteins. It was found CD47 protein was still retained on RBCs@Hb@GOx NPs (Fig.S7), validating the successful coating of erythrocyte membrane.

\subsection{Evaluation of Catalytic Performance of RBC@ Hb@GOx NPs}

The Fenton catalytic performance of the RBC@Hb@GOx NPs was evaluated [45, 46]. The RBC@Hb@GOx NPs were incubated with glucose solution $(10 \mathrm{mM})$ to start cascade Fenton-type reactions (Figs. 3a and S8). Amplex red, a fluorescence probe that can respond to $\mathrm{H}_{2} \mathrm{O}_{2}$ with fluorescence intensity changes, was applied to measure the generated $\mathrm{H}_{2} \mathrm{O}_{2}$ during the catalytic process. As presented in Fig. 3b, $\mathrm{c}$, the fluorescence intensity of Amplex red increased significantly with the reaction time, indicating the efficient production of $\mathrm{H}_{2} \mathrm{O}_{2}$. Almost no $\mathrm{H}_{2} \mathrm{O}_{2}$ were observed in control groups (Fig. 3d), suggesting that the $\mathrm{H}_{2} \mathrm{O}_{2}$ was produced by GOx-medicated glucose oxidation. To detect the production of free radicals, 1,3-diphenylisobenzofuran (DPBF) was employed to quantitatively analyze $\cdot \mathrm{OH}$ [47]. The generated - $\mathrm{OH}$ can oxidize DPBF and result in the reduction in the absorption intensity at wavelength of $410 \mathrm{~nm}$ in UV-Vis absorption spectrum. In Fig. 3e, the absorption at $410 \mathrm{~nm}$ of 
(a)
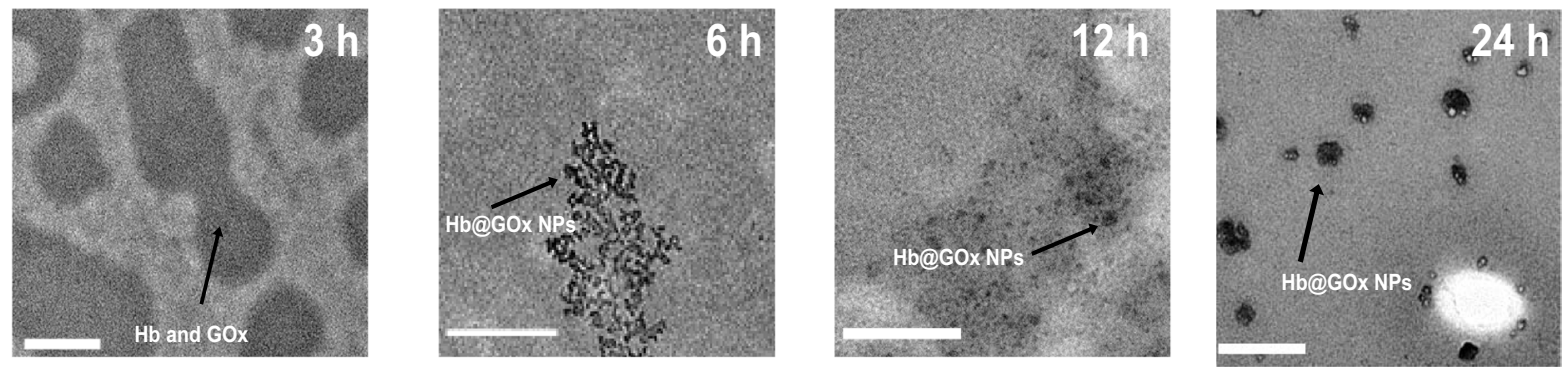

(b)
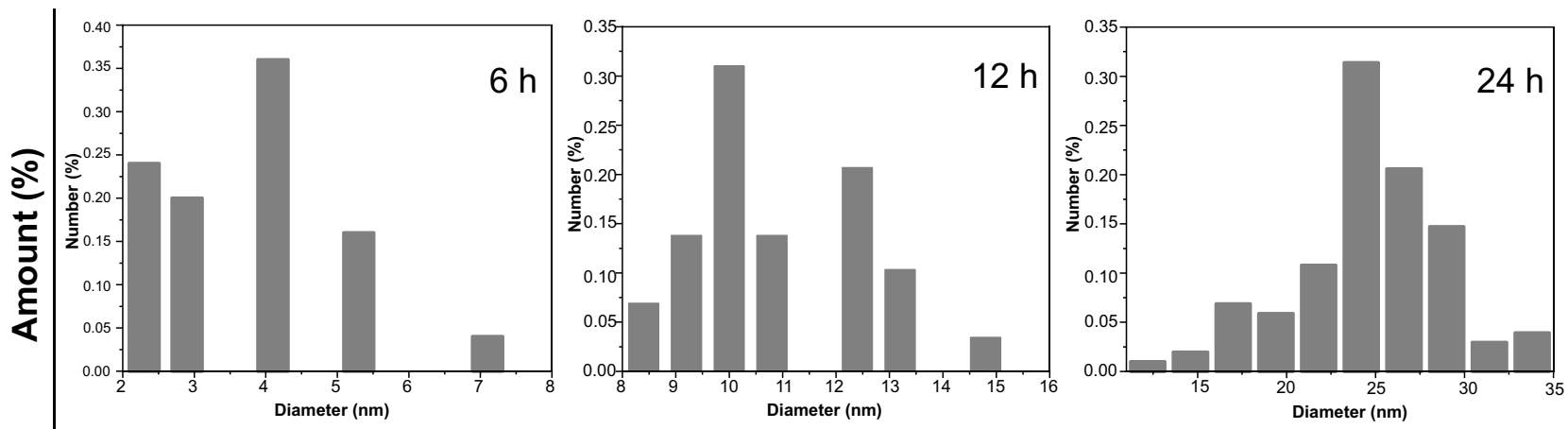

(c)

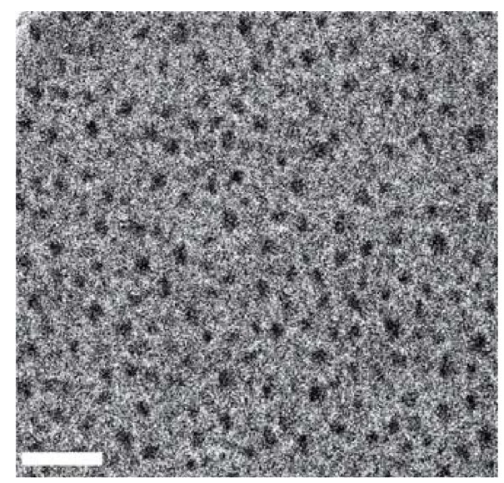

(f)

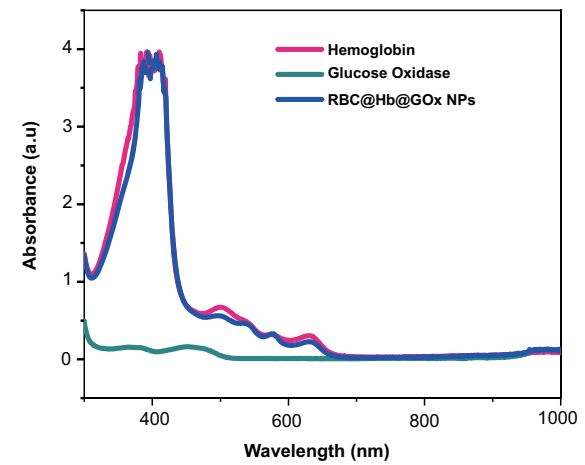

(d)

\section{Sized by TEM}

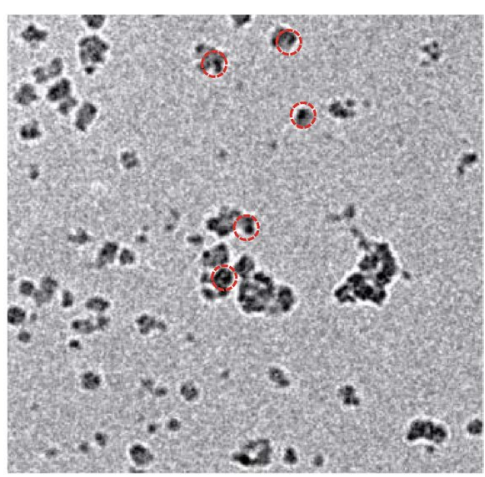

(g)

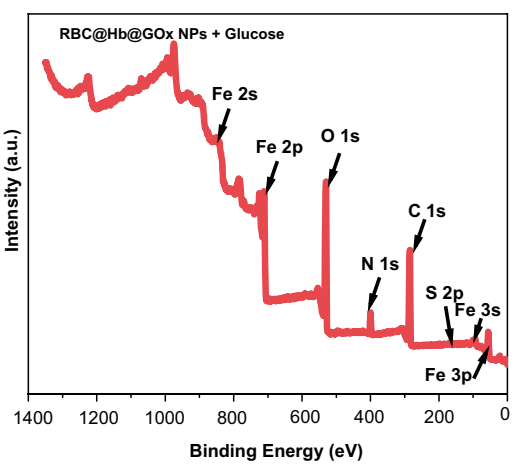

(e)

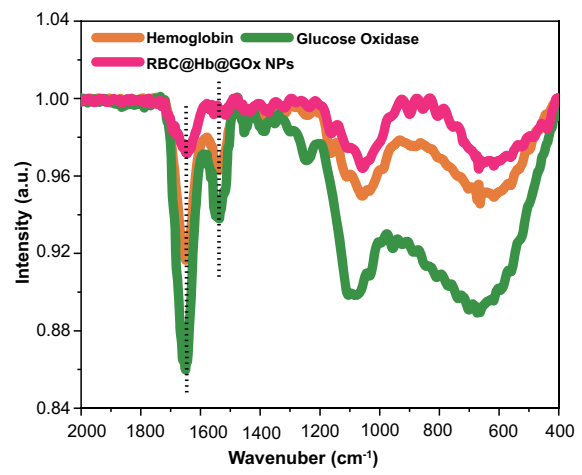

(h)

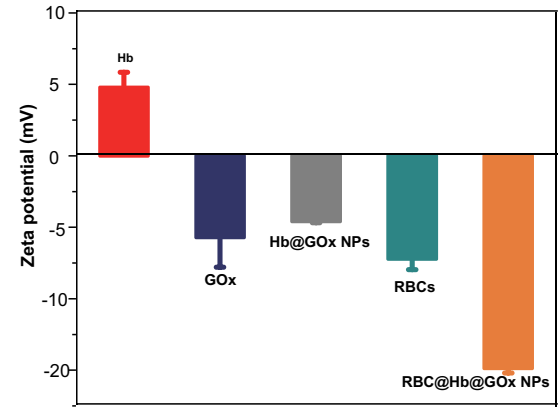

Fig. 2 Characterization of as-fabricated Hb@GOx NPs and RBC@Hb@GOx NPs. a TEM images of as-fabricated Hb@GOx NPs at each reaction time. b Size of Hb@GOx NPs at each reaction time measured by TEM. Scale bar: $100 \mathrm{~nm}$. TEM images of $\mathbf{c}$ Hb@GOx NPs (scale bar: $100 \mathrm{~nm}$ ) and d RBC@ Hb@GOx NPs (scale bar: 100 nm).e FT-IR spectra of Hb, GOx and RBC@Hb@GOx NPs. f UV-Vis absorption spectra of free Hb, GOx and RBC@Hb@GOx NPs. g XPS of RBC@Hb@GOx NPs. h Zeta potentials of the Hb, GOx, RBC membranes, Hb@GOx NPs and RBC@Hb@GOx NPs. Data are shown as mean \pm SD $(n=3)$ 
DPBF decreases obviously after 25 min reaction. The results provided a clear evidence that the bioactivities of the GOx and $\mathrm{Hb}$ were not affected after the crosslinking reactions.

Since electron spin resonance (ESR) spectrum has been considered as the most effective assay to detect the generation of ROS [48]. We selected 5-tertbutoxycarbonyl-5-methy1-pyrroline N-oxide (BMPO), the traditional ROS trapping agents, to confirm the generation of $\cdot \mathrm{OH}$ and $\mathrm{O}_{2}^{--}$. As shown in Fig. 3f, g, both Hb@GOx NPs and RBC@Hb@GOx NPs triggered the Fenton reactions in glucose solution $(10 \mathrm{mM})$ and resulted in two typical spectra. The four-peak spectrum with an intensity ratio of 1:2:2:1 belonged to the BMPO/OH adduct, while the spectrum with an intensity ratio of $1: 1: 1: 1$ showed adduct of BMPO/O-- Thus, as-fabricated NPs had excellent performance to produce $\cdot \mathrm{OH}$ and $\mathrm{O}_{2}^{--}$.

We then studied the change in $\mathrm{pH}$ values during the catalytic reaction. As shown in Fig. 3h, i, for RBC@Hb@ GOx NPs incubated with glucose, the $\mathrm{pH}$ value continually decreased from 6.20 to 3.19 within 45 min due to the generation of gluconic acid. The experimental results further validated that GOx's remained original activity remained after crosslinking with $\mathrm{Hb}$. It is worth noting that there are synergic effects by combining $\mathrm{Hb}$ and GOx for Fenton reactions. It is well known that TME is hypoxic, which limits the performance of GOx since the oxygen is critical in catalyzing glucose into $\mathrm{H}_{2} \mathrm{O}_{2}$. This problem was perfectly overcome by $\mathrm{Hb}$, which is an oxygen-carrying protein. The oxygen-carrying capability of $\mathrm{Hb}$ was investigated in Fig. S9, showing that the dissolved oxygen in RBC@Hb@GOx solution increased gradually with time. In short, our results suggested that the RBC@Hb@GOx NPs could be utilized as a powerful CDT nanoagent.

\subsection{Evaluation of In Vitro Cytotoxicity of RBC@Hb@ GOx NPs}

To explore the in vitro cytotoxicity of RBC@ $\mathrm{Hb} @ \mathrm{GOx}$ NPs, methyl thiazolyl tetrazolium (MTT) assays were conducted [49]. U87MG and NHI3T3 cells were incubated with RBC@Hb@GOx NPs with concentrations ranging from 0 to 1000 ug mL $\mathrm{mL}^{-1}$ (Fig. 4a). RBC@Hb@GOx NPs showed negligible cytotoxicity in the cell lines, even at the concentration as high as $1000 \mathrm{ug} \mathrm{mL}^{-1}$. In addition, the acquired hemolysis ratio was much less than $1 \%$ when the concentration of RBC@Hb@GOx NPs reached $2500 \mu \mathrm{g} \mathrm{mL} \mathrm{L}^{-1}$ (Fig. S10). Moreover, the degradation of RBC@Hb@GOx NPs was also investigated via UV-Vis absorption spectrum (Fig. S11). The NPs completely degraded after $30 \mathrm{~min}$ in glucose solution. These results indicated that the nanoparticles possess excellent biocompatibility and biodegradability. When the glucose was added to a mixture of cells and RBC@ $\mathrm{Hb} @$ GOx NPs, the NPs were activated. High toxicity to the cells was observed as a result of ROS-caused oxidative damage (Fig. 4b). In comparison, adding glucose to $\mathrm{Hb}$ solution did not have a marked effect on the viability due to the lack of GOx to convert glucose to $\mathrm{H}_{2} \mathrm{O}_{2}$, while adding $\mathrm{H}_{2} \mathrm{O}_{2}$ directly to $\mathrm{Hb}$ solution only showed marginal cytotoxicity. These findings suggested that GOx played an important role in enhancing the Fenton reactions. The Calcein-AM/ PI staining assay [50] was performed to further evaluate the anticancer effects of RBC@Hb@GOx NPs (Fig. 4c). The dead or alive cells were differentiated by the fluorescence color (red fluorescent spots for dead cells, green spots for living cells). It can be seen that in the presence of glucose, the Hb@GOx NPs and RBC@Hb@GOx NPs showed the highest red/green ratio, indicating the excellent capability of inhibiting the growth of cancer U87MG cells.

To quantify the intracellular ROS generated by the RBC@ Hb@GOx NPs, 2'-7'-dichlorofuorescin diacetate (DCFHDA) assay was used [51] which converted non-fluorescent DCFH-DA into fluorescent 2'-7'-dichlorofuorescin (DCF) when oxidized by ROS. In Fig. 4d, no obvious green fluorescence was observed in U87MG cells for the PBS group and Hb@GOx NPs or RBC@Hb@GOx NPs in the absence of glucose, suggesting insignificant ROS production. Notably, groups Hb@GOx NPs or RBC@Hb@GOx NPs with glucose induced a large amount of intracellular ROS as exhibited by the strong green fluorescence emission of DCF. Furthermore, the intracellular ROS level was determined via flow cytometry experiment. As shown in Fig. 4e, the fluorescent intensity of U87MG cells incubated with $\mathrm{Hb} @$ GOx NPs (33.57\%) or RBC@Hb@GOx NPs (35.36\%) in the presence of glucose was stronger than PBS group (4.38\%), which indicated that both Hb@GOx NPs and RBC@Hb@ GOx NPs were highly efficient in generating ROS. Similar amount of ROS was generated from Hb@GOx NPs and RBC@Hb@GOx NPs, meaning that the modification of the RBC membrane had a negligible effect on the catalytic performance. Together with the ESR experimental results, it can be concluded that in the presence of glucose, RBC@ $\mathrm{Hb} @$ GOx NPs could be able to produce massive intracellular ROS to kill tumor cells. 
(a)

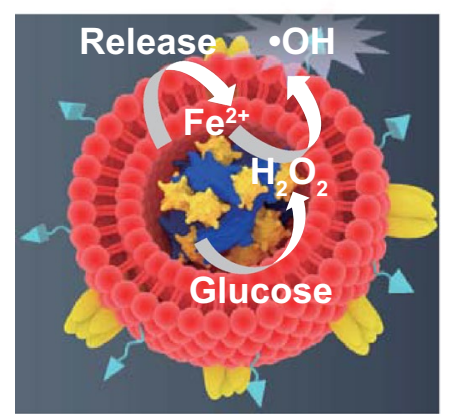

(d)

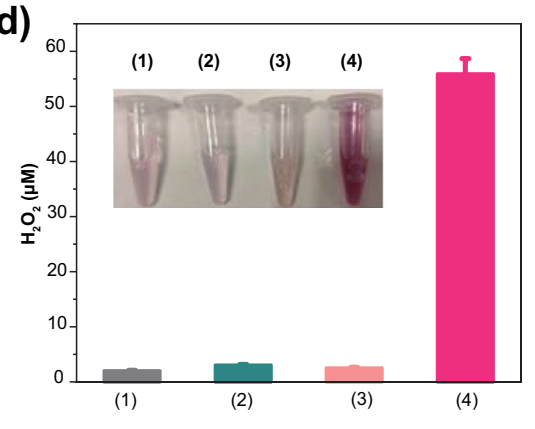

(g)

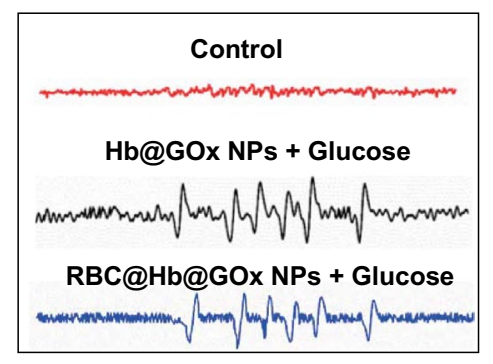

(b)

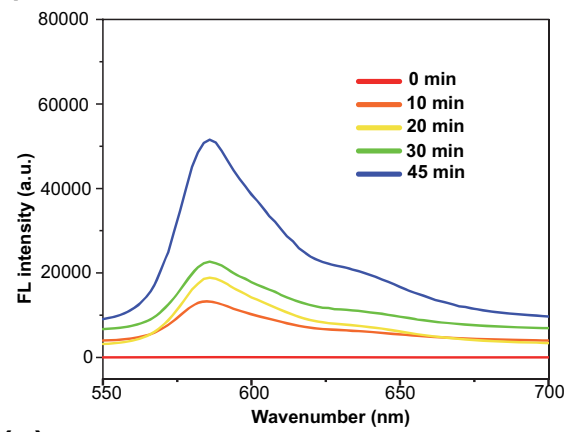

(e)

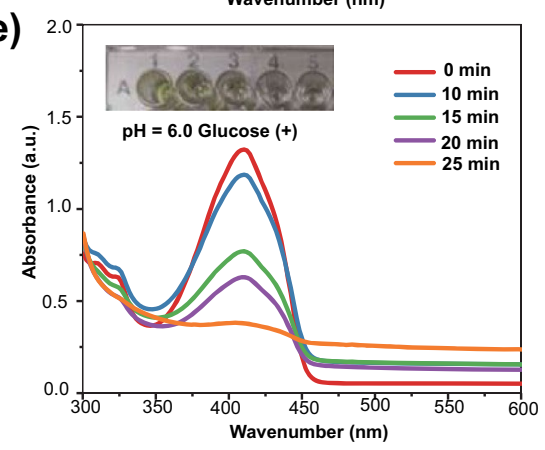

(h)

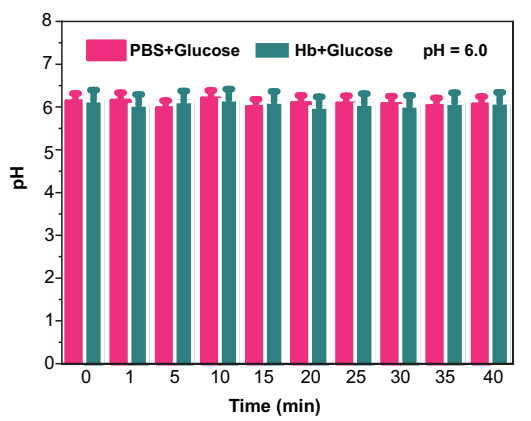

(c)

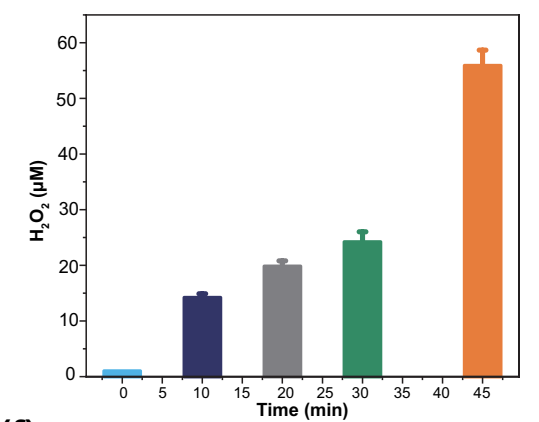

(f)

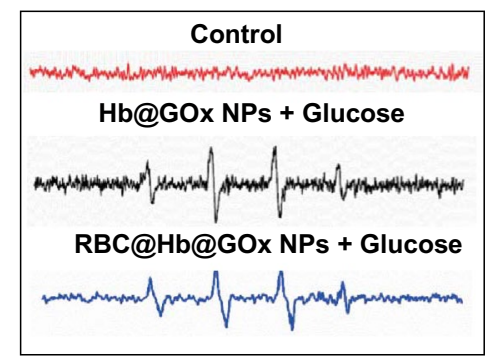

(i)

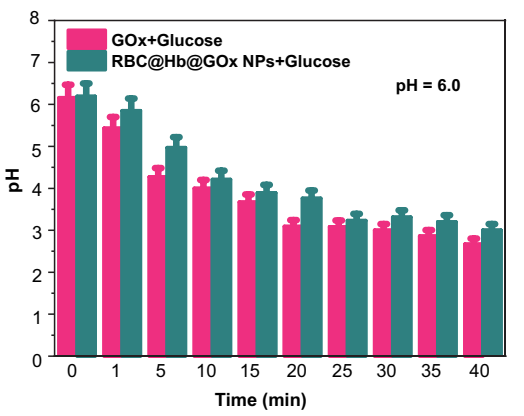

Fig. 3 Verification of $\mathrm{H}_{2} \mathrm{O}_{2}$ and ROS $\left(\cdot \mathrm{OH}, \mathrm{O}_{2}^{-}\right)$generation in vitro. a Synergism schematic of Fenton reaction of RBC@Hb@GOx NPs triggered by tumor microenvironment. b Fluorescence spectra of Amplex red probe incubated with RBC@Hb@GOx NPs in the presence 10 mM of glucose. c Production of $\mathrm{H}_{2} \mathrm{O}_{2}$ during the catalytic reactions. d Amplex red probe incubated with different groups (PBS, Hb, RBC@Hb@GOx NPs without glucose, and RBC@Hb@GOx NPs with glucose). e UV-Vis absorption spectrum of DPBF. f, g ESR spectra of BMPO after incubation of Hb@GOx NPs and RBC@Hb@GOx NPs with glucose $(10 \mathrm{mM})$. h pH values of PBS, Hb in $10 \mathrm{mM}$ of glucose. i pH values of Hb and GOx, and RBC@Hb@GOx NPs in 10 mM of glucose. Data are shown as mean $\pm \mathrm{SD}(n=3)$

\subsection{Evaluation of In Vitro Mitochondrial and Endoplasmic Reticulum Damage}

We further investigated the mechanisms of ROS-mediated cell death. Mitochondria are central organelles and play a critical role in apoptosis in reacting to stress. Many factors induce cell apoptosis, including loss of mitochondrial membrane potential, release of cytochrome c (Cyto $c)$, and caspase-activating protein. In the cell apoptosis progress, the loss of mitochondrial membrane potential is normally a signal of early mitochondrial damage, which can be investigated by JC-1 staining assay. It has been proved that JC-1 is an ideal fluorescent probe to detect mitochondrial membrane potential $(\Delta \Psi m)$. When the mitochondrial membrane potential is high (normal mitochondria), JC-1 dyes aggregate in the mitochondrial matrix to form polymers called JC-1 
(a)

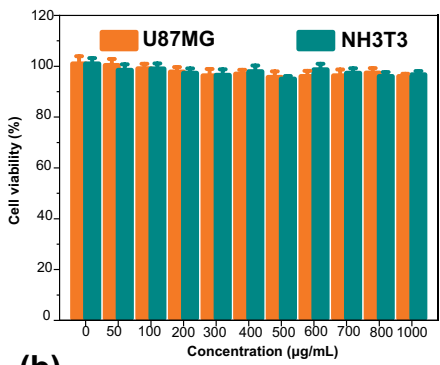

(b)

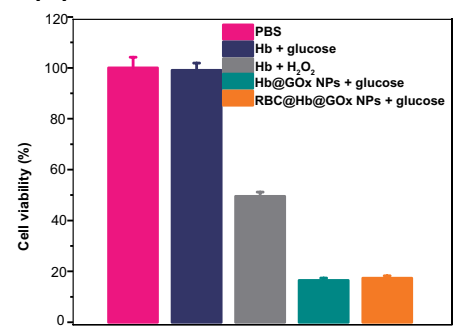

(d)
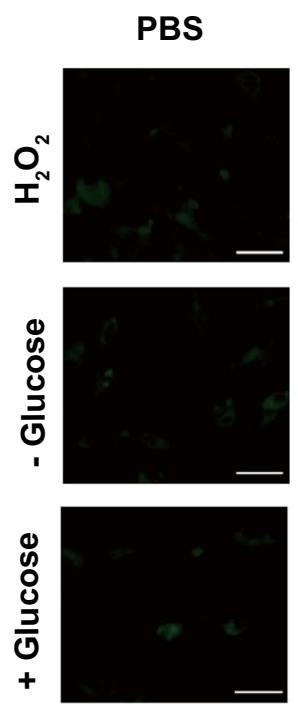

(c)
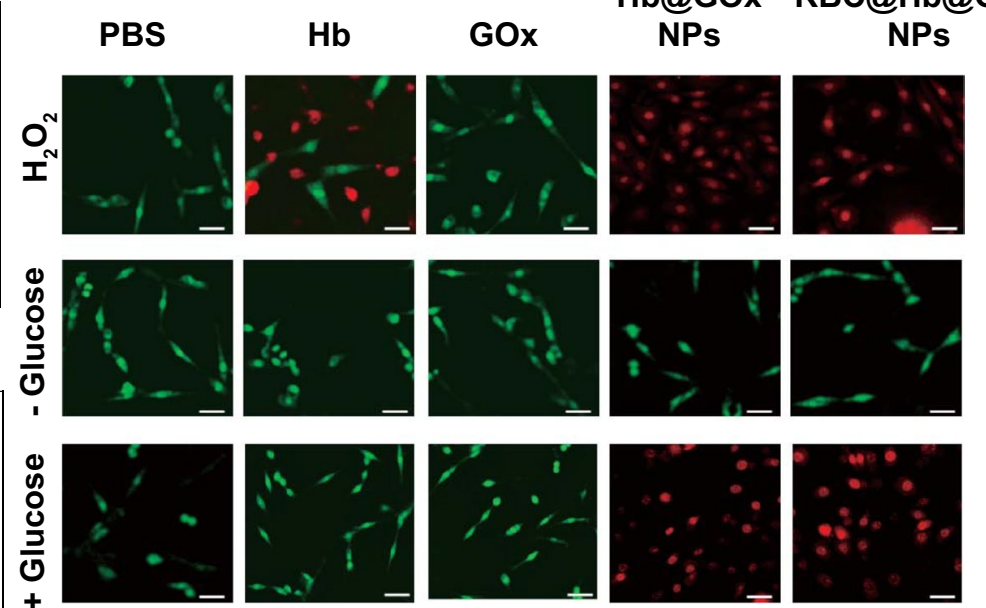

Hb@GOx RBC@Hb@GOx

$\mathrm{Hb}$

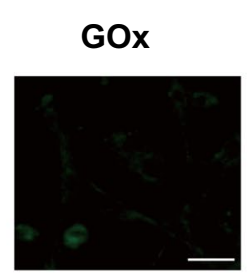

NPs
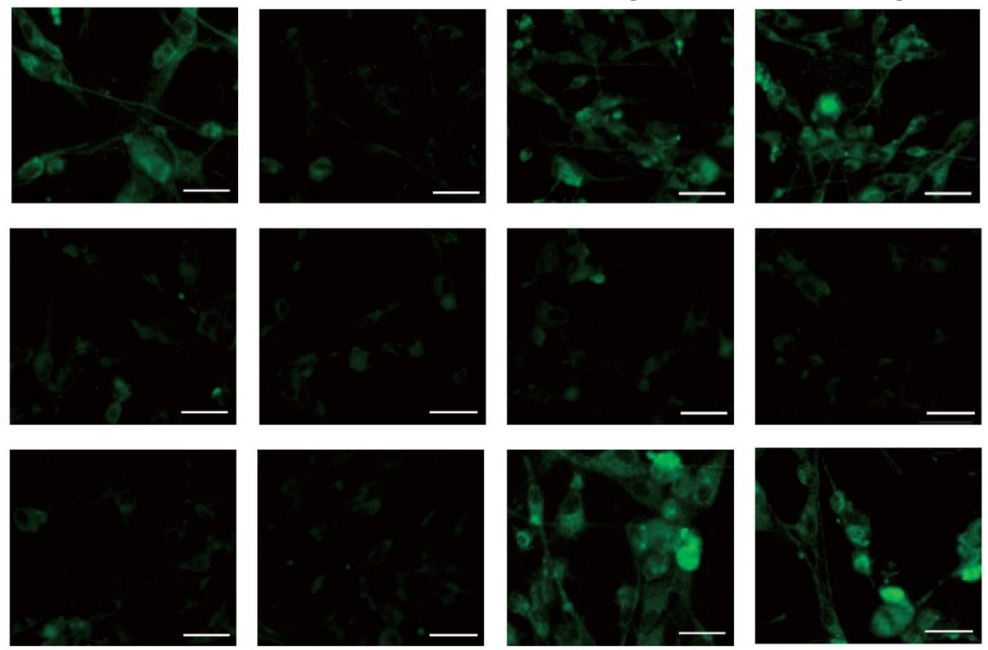

(e)
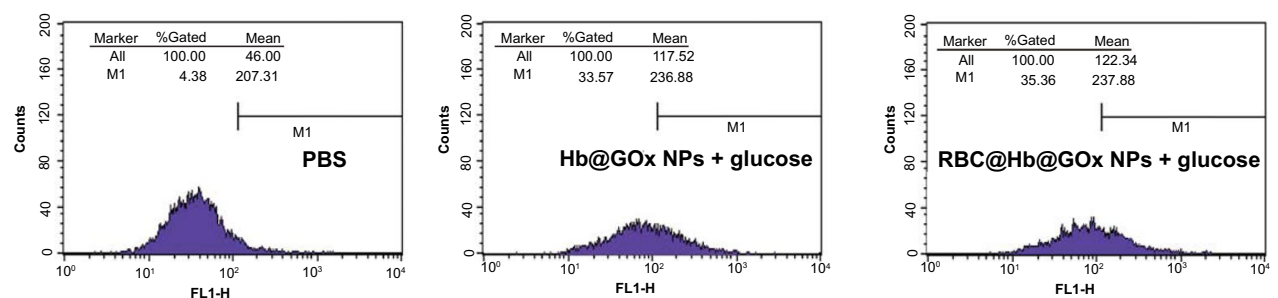

Fig. 4 In vitro cytotoxicity and intracellular catalytic mechanism. a Cytotoxicity of RBC@Hb@GOx NPs to U87MG and NIH3T3 cells without glucose. b Cell viability assay of (1) PBS, (2) $\mathrm{Hb}$ and glucose (10 mM), (3) GOx and glucose, (4) $\mathrm{Hb}^{\text {and }} \mathrm{H}_{2} \mathrm{O}_{2}$, (5) GOx and $\mathrm{H}_{2} \mathrm{O}_{2}$, (6) $\mathrm{Hb} @$ GOx NPs and glucose, and (7) RBC@Hb@GOx NPs and glucose-treated U87MG cells. $\mathbf{c}$ Live/dead assay of U87MG cells after incubation with different samples for $24 \mathrm{~h}$ and subsequent staining with AM/PI probe. Scale bar: $100 \mu \mathrm{m}$. d Detection of intracellular ROS after incubating U87MG with different samples. Scale bar: $50 \mu \mathrm{m}$. e The level of intracellular ROS was measured by flow cytometry using the peroxide-sensitive fluorescent probe DCFH-DA. Data are shown as mean $\pm \mathrm{SD}(n=3)$ 
aggregates, producing red fluorescence. While JC-1 aggregates are release from mitochondria matrix to cytoplasm to form JC-1 monomer, corresponding fluorescence transits from red to green, indicating that the mitochondrial membrane potential is low (damaged mitochondria) (Fig. S12). Thus, it is very convenient to detect mitochondrial membrane potential through observing the changes of fluorescent color and the relative ratio of red and green fluorescence $[52,53]$. In this work, UMG87 cells incubated with different samples were stained with JC-1 dyes and analyzed via flow cytometry. As shown in Fig. 5a, b, the red fluorescence (normal mitochondria) of UMG87 cells in three groups including PBS, $\mathrm{Hb}$, and GOx was observed. In contrast, the increase in green fluorescence intensity (damaged mitochondria) was obviously seen in groups treated with Hb@GOx NPs and RBC@Hb@GOx NPs with glucose (10 mM) after 20 min incubation. The Flow cytometry plots in Fig. 5c shows the percentage of mitochondrial membrane depolarized cells increased from 7.38\% (control group) to 35.18\% (Hb@GOx NPs with 10 mM glucose) and 32.91\% (RBC@Hb@GOx NPs with $10 \mathrm{mM}$ glucose), respectively.

As mentioned in previous studies, calreticulin (CRT) is rich in endoplasmic reticulum. Normally, ROS-based endoplasmic reticulum stress would induce the CRT transfer to the surface of plasma membrane (Fig. S13a). Besides, high mobility group protein B1 (HMGB1), as a highly conserved nuclear protein, is also released from the nuclear while the cells are dying [54]. Therefore, the CRT exposure and HMGB1 release were assessed to evaluate the intracellular oxidative stress in UMG87 cells. We evaluated the CRT exposure in UMG87 cells after incubating with PBS, Hb, GOx, Hb@GOx NPs, and RBC@Hb@GOx NPs (all groups treated with glucose solution, $10 \mathrm{mM}$ ) via immunofluorescence staining and CLSM analysis. As displayed in Fig. 5d, the largest number of CRT staining cells was detected in the Hb@GOx NPs and RBC@Hb@GOx NPs groups, indicating that these two groups could significantly induce the CRT translocation from the endoplasmic reticulum to cell surface due to the large amount of ROS generation. Next, the increased HMGB1 release in cell culture supernatant from the Hb@GOx NPs and RBC@Hb@GOx NPs-treated UMG87 cells was also detected by western blot analysis (Fig. S13b). The above findings revealed that the intracellular ROS generated by RBC@Hb@GOx NPs caused mitochondria damage and led to the eventual cell apoptosis.

\subsection{Evaluation the Biodistribution of RBC@Hb@GOx NPs In Vivo}

Orthotopic U87MG gliomas (without fluorescence) bearing mice were constructed as animal models. ICG-loaded RBC@Hb@GOx NPs were injected intravenously to investigate their ability to cross the BBB (Figs. 6a and S14) [27]. In this study, we employed a passive targeting strategy. As shown in Fig. 6b, the fluorescence at the tumor site was increased after $12 \mathrm{~h}$ post-injection with RBC@Hb@GOx NPs. Interestingly, it maintained strong fluorescence signals even after $72 \mathrm{~h}$. The ex vivo fluorescence images of major organs and brain (corresponding fluorescence intensity is shown in Fig. S15) were obtained at $72 \mathrm{~h}$ (Fig. 6c). There was an obvious fluorescence signal in the brain, while no apparent fluorescence could be observed in the other organs. Combing the above results with the fluorescence intensity at each time point (Fig. 6d) and staining experiments of the slice from different organs (Fig. S16), we could conclude that the RBC@Hb@GOx NPs crossed the BBB and exhibited notable tumor accumulation and prolonged retention properties. Compared to receptor-mediated transcytosis, the delivery efficiency was significantly increased.

To further investigate the role of RBC membrane in crossing the BBB, we synthesized ICG-loaded RBC@Hb@GOx NPs and Hb@GOx NPs to compare their accumulation in tumors (Fig. 6e). From Fig. 6f, we found that the ICG-Hb@ GOx NPs-treated group also exhibited strong fluorescence after $36 \mathrm{~h}$ post-injection, whereas the fluorescence intensity in the ICG-RBC@Hb@GOx NPs-treated group was considerably higher (Fig. S17a, b). The results showed that the RBC membrane was not the essential component but rather a facilitator in $\mathrm{BBB}$ crossing. This might be because that the RBCs membrane improved the circulation time and made the NPs softer and easier to deform, providing higher chances for the NPs to transverse the BBB. Similarly, the fluorescence imaging and intensity for major organs were tested after $72 \mathrm{~h}$ post-injection (Figs. $6 \mathrm{~g}$ and $\mathrm{S} 17 \mathrm{c}$ ) and TUNEL staining from different organs (Fig. S18), showing that obvious fluorescence signals could be observed in both two groups. Therefore, we believe that in this case, the long circulating time and the small size are the contributing factors for the NPs to cross BBB and accumulate at the tumor site. 
(a)
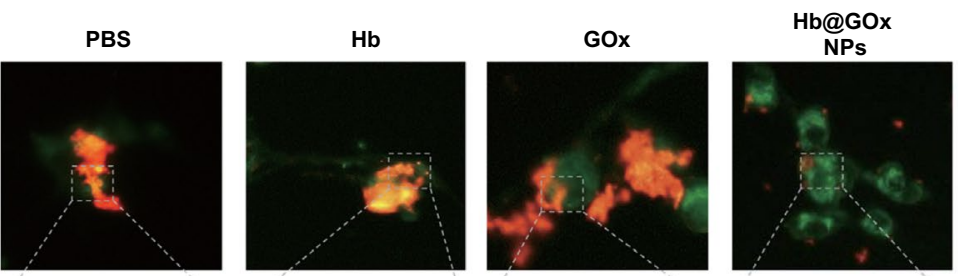

RBC@Hb@GOX

(b)
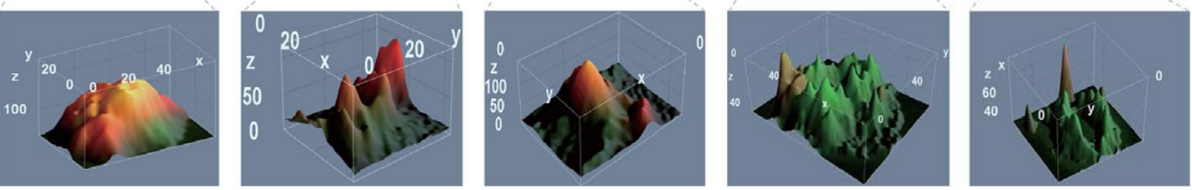

(c)
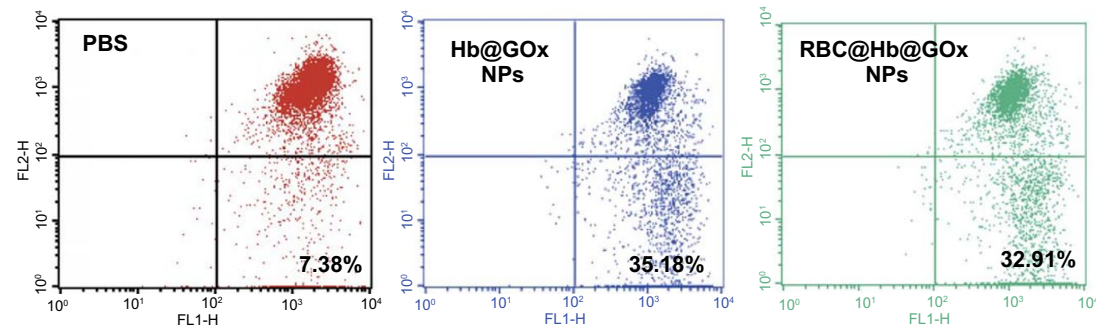

(d)
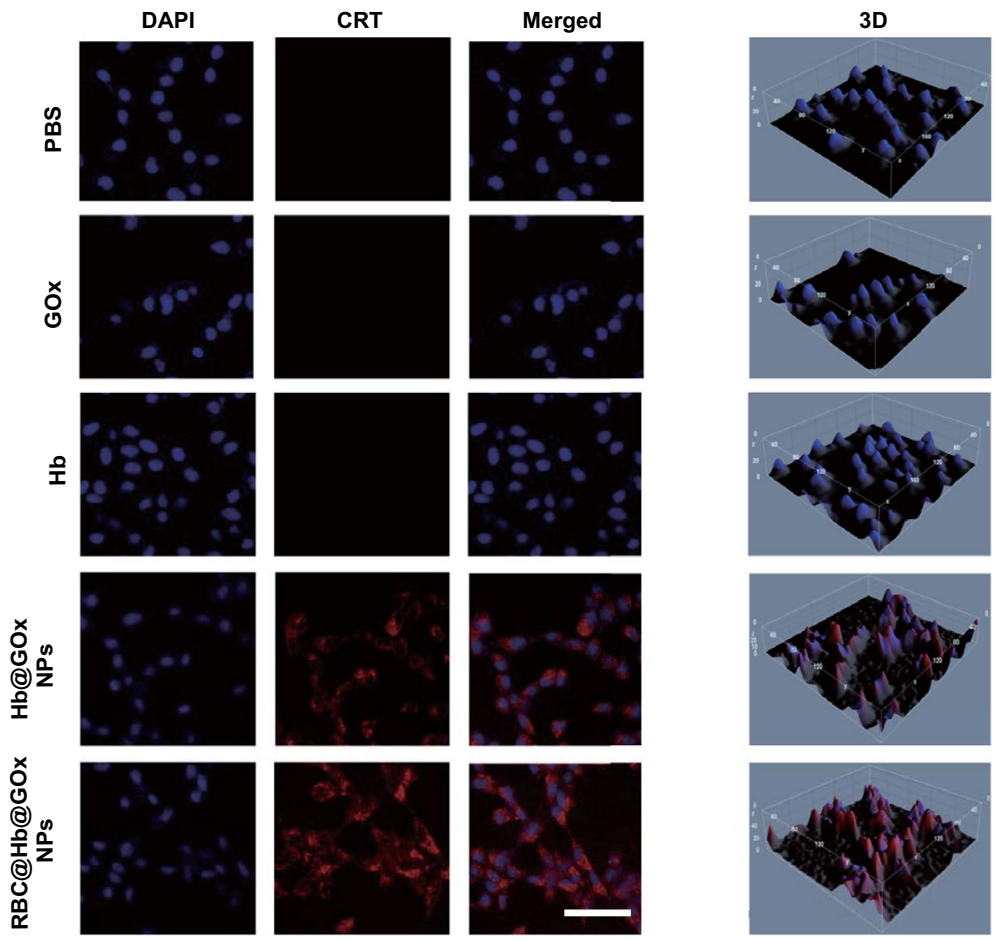

Fig. 5 Mitochondrial and endoplasmic reticulum damage induced by RBC@Hb@GOx NPs. a CLSM images of JC-1 stained U87MG cells after different treatments. The fluorescence transition from red to green indicated significant mitochondrial damage. Scale bar: $50 \mu \mathrm{m}$. b 3D images of JC-1 stained U87MG cells after different treatments. $\mathbf{c}$ Evaluation of mitochondrial potential changes via flow cytometer test after different treatments. d Evaluation of CRT transferring via CRT staining assay after different treatments. Scale bar: $50 \mu \mathrm{m}$ 
(a)

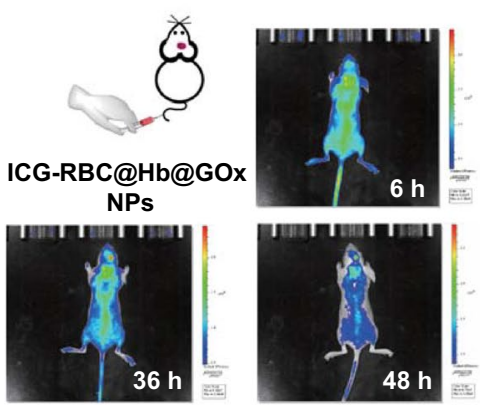

(e)

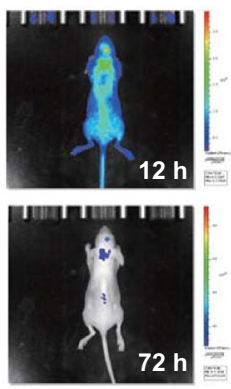

(1)

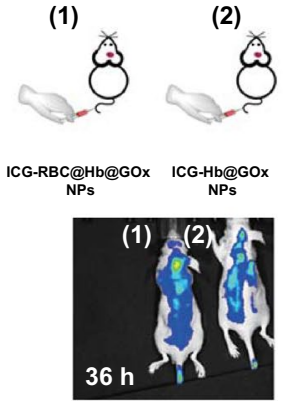

(f)

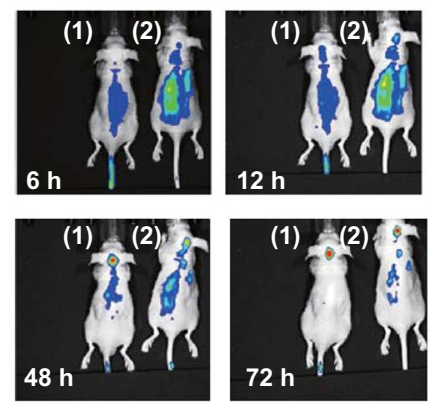

(c)

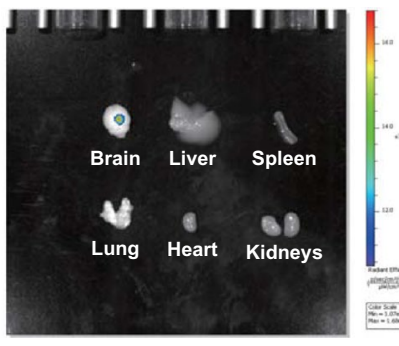

(d)

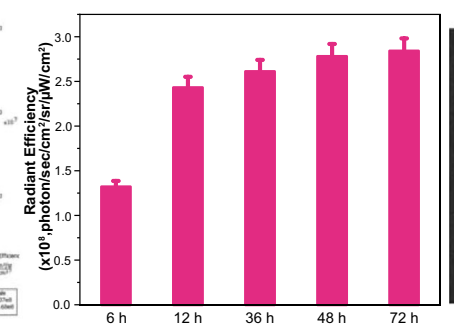

(g)

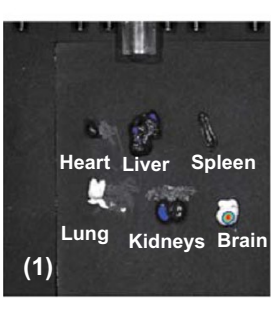

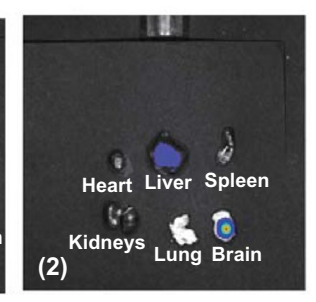

Fig. 6 In vivo fluorescence imaging of orthotopic U87MG tumor-bearing mice for evaluating the transportability across the BBB. a Schematic illustration of injection of ICG-RBC@Hb@GOx NPs to orthotopic U87MG tumor-bearing mice. b Real-time fluorescence imaging of tumorbearing mice after intravenous injection of ICG-RBC@Hb@GOx NPs. c Ex vivo fluorescent images of major organs and tumors at 72 h postinjection. d Semiquantitative fluorescence analysis in the tumor site at different time points after injection of ICG-RBC@Hb@GOx NPs. e Schematic illustration of injection of ICG-RBC@Hb@GOx and ICG-Hb@GOx NPs NPs to orthotopic U87MG tumor-bearing mice. f Realtime fluorescence imaging of tumor-bearing mice after intravenous injection of ICG-RBC@Hb@GOx NPs and ICG-Hb@GOx NPs. g Ex vivo fluorescent images. Data are shown as mean $\pm \operatorname{SD}(n=3)$

\subsection{Evaluation of In Vivo CDT of Orthotopic GBM Tumor}

Encouraged by the satisfactory BBB transportability in vivo, we further investigated the therapeutic efficacy of RBC@ Hb@GOx NPs. The mice bearing orthotopic GBM tumor with self-fluorescence were constructed. IVIS ${ }^{\circledR}$ imaging system was used to track the tumor response to treatment. Firstly, we did a short-term survival experiment in mice (Fig. S19a). PBS (control group), GOx, and RBC@Hb@ GOx NPs were administrated by tail vein injection to evaluate the therapeutic performances, respectively. As shown in Fig. S19b, during 15-day treatment, the fluorescence intensity in control and GOx groups showed significant increase, indicating that no therapeutic effect on the brain tumor. In contrast, for the group treated with RBC@Hb@GOx NPs, fluorescence intensity increased slowly and the area with fluorescence was much smaller compared to the other two groups, which implied that the RBC@Hb@GOx NPs was able to suppress the tumor growth. The therapeutic effect was further confirmed by H\&E and Congo red staining (Fig. S19c). These staining images indicated an extensive cell apoptosis in GBM in the RBC@Hb@GOx NPs-treated group. Besides, the whole brain was also sliced from mice after 15 days (Fig. S20a). It was apparent that the satisfactory tumor suppression effects came from the highly toxic hydroxyl radicals produced by the sequential biological/ chemical-catalytic reactions by the RBC@Hb@GOx NPs. The as-produced toxic hydroxyl radicals then killed the cancer cells in a mitochondria-mediated apoptosis pathway.

A long-term survival experiment was conducted in mice to compare the therapeutic effect of Hb@GOx NPs and RBC@Hb@GOx NPs as outlined in Fig. 7a. It is clearly seen from Fig.7b that both Hb@GOx NPs- and RBC@Hb@ GOx NPs-treated groups significantly suppressed the growth of U87MG glioblastoma tumor. The tumor suppression efficiency was quantified by measuring the changes of the fluorescence intensity at the tumor sites. As presented in Fig. 
(a)

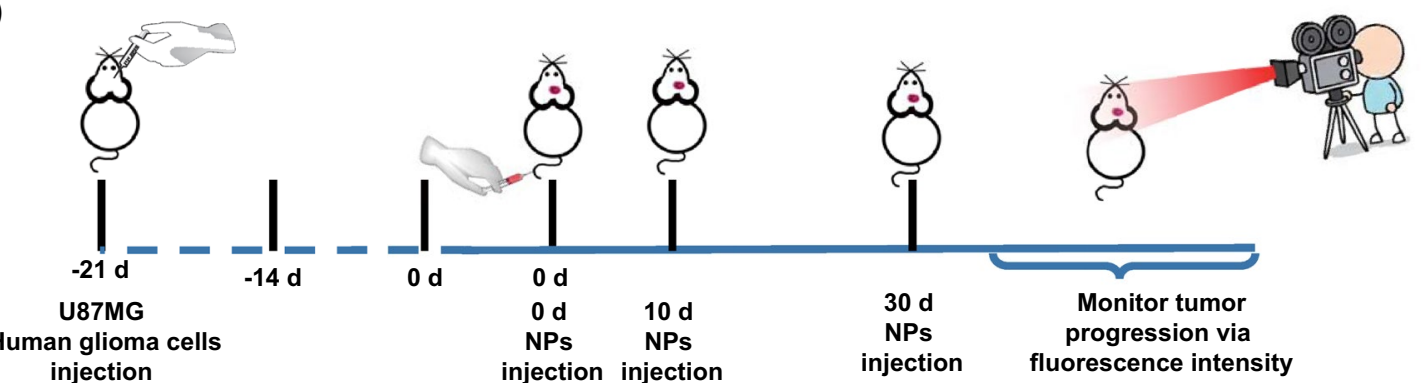

(b)
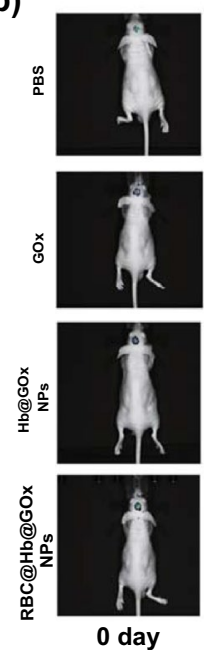

(c)

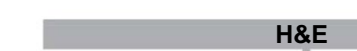

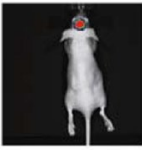
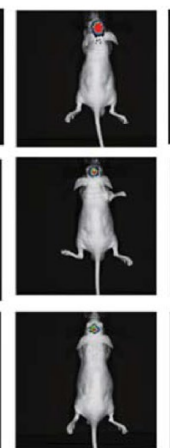

7 days
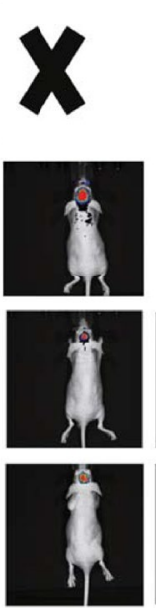

15 days
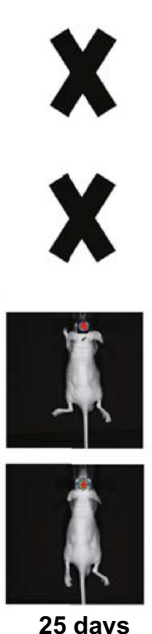
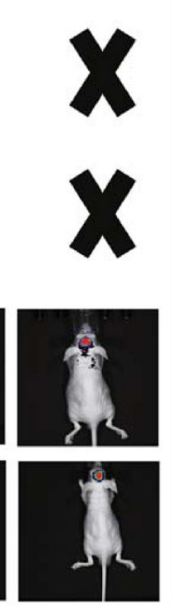

35 days (d)

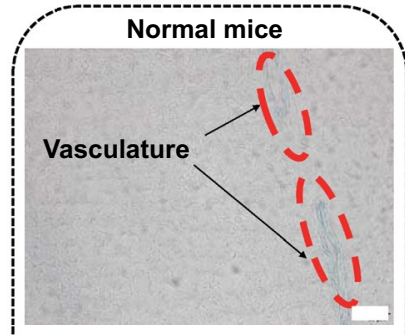

Orthotopic U87MG tumor mice

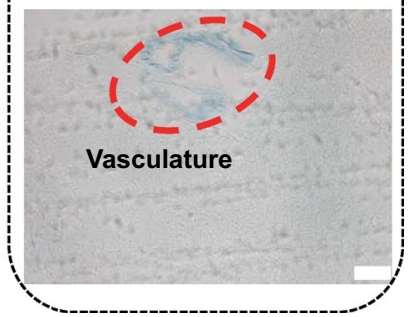

TUNEL
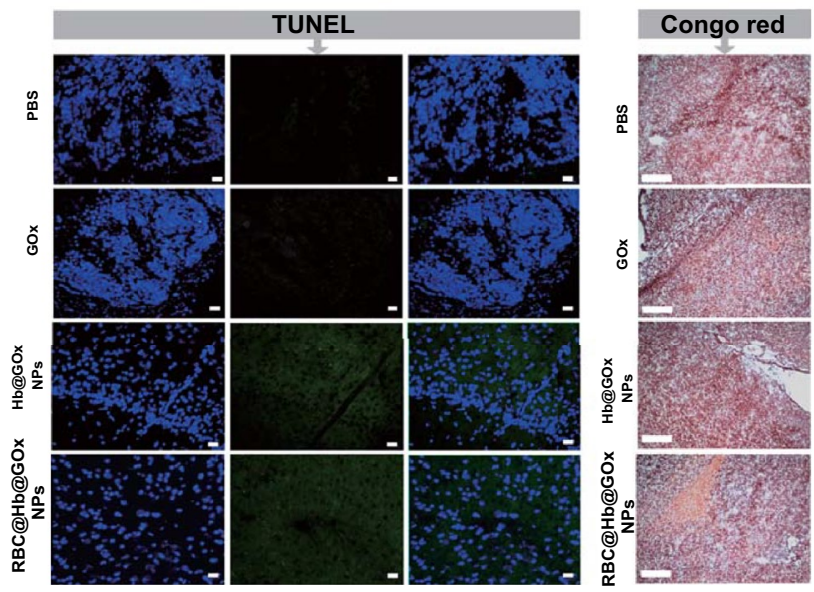

Fig.7 In vivo therapeutic efficacy of Hb@GOx NPs and RBC@Hb@GOx NPs in orthotopic glioma-bearing mice. a Schematic illustration of long-term survival experiment in orthotopic U87MG tumor-bearing mice with PBS, GOx, Hb@GOx NPs, and RBC@Hb@GOx NPs injection. b Representative bioluminescent images of orthotopic U87MG tumor-bearing mice in different groups in 35-day treatments. $\mathbf{c}$ Orthotopic U87MG tumor in the brain after H\&E (scale bar: $50 \mu \mathrm{m}$ ), TUNEL (scale bar: $20 \mu \mathrm{m}$ ), and Congo red staining (scale bar: $50 \mu \mathrm{m}$ ) after treatments. d EB staining of brain sections after treatment. Scale bar: $50 \mu \mathrm{m}$

S20b, quantitative analysis showed that when treated with PBS or GOx, the orthotopic brain tumor grew rapidly. However, the tumor treated with Hb@GOx NPs and RBC@Hb@ GOx NPs maintained relatively constant size. As shown in
Fig. 7c, the H\&E staining experiments of brains tumor tissue were obtained from control group (PBS)-treated mice and mice co-treated with GOx, Hb@GOx NPs, and RBC@Hb@ GOx NPs. In control group, the H\&E staining experiments 
presented large tumor cell populations with higher instance of actively dividing nuclear morphology. For the treated groups, H\&E stains shown that there was an obvious area of dead cells with radial propagations in orthotopic U87MG tumor. These results were in close agreement with TUNEL staining and Congo red staining. Finally, typical Evans blue (EB) stained brains in Fig. 7d showed that both normal mice and orthotopic U87MG tumor-bearing mice maintained an intact structure of BBB, because the EB could only be detected in brain vascular, further validating that our NPs possessed an excellent transportability of BBB. Finally, the slight changes in body weight (Fig. S21a) and the survival rate (Fig. S21b) indicated that RBC@Hb@GOx NPs possessed a remarkable biocompatibility.

Besides, the blood biochemical data, including red blood cells (RBC), white blood cells (WBC), hemoglobin (HGB), hematocrit (HCT), mean corpuscular volume (MCV), mean corpuscular hemoglobin concentration (MCHC), platelets (PLT), and mean corpuscular hemoglobin (MCH), liver and kidney function test including alanine transaminase (ALT), aspartate transaminase (AST), albumin (ALB), creatinine (CREA), blood urea nitrogen (BU), and uric acid (UA) were applied to evaluate the in vivo toxicity of RBC@Hb@GOx NPs (Fig. S22). There was no apparent discrepancy can be observed from all groups, what is more, no significant pathological changes of the major organs from all experimental groups were observed by H\&E stained organ slices (Fig. S23), demonstrating the excellent biocompatibility of RBC@ Hb@GOx NPs. These results showed that the RBC@Hb@ GOx NPs as a powerful CDT nanoagent could efficiently inhibit the growth GBM and elongate the survival time.

\section{Conclusions}

In summary, we developed a new type of CDT nanoagent for GBM treatment. The natural $\mathrm{Hb}$ and GOx were employed as highly efficient Fenton catalysts. The excellent biocompatibility and biodegradability greatly reduced the toxicity of the nanomaterials and improved the safety of CDT. With facile assembling and crosslinking techniques, $\mathrm{Hb}$ and GOx were formulated into protein superstructures. They could be utilized as self-delivery entities, thereby eliminating the need of conventional drug carriers. RBC membranes were coated on the protein superstructures to reduce the immunogenicity and enhance the circulation time, which facilitated the delivery of the NPs to cross BBB/BTB. We showed that the RBC@Hb@GOx NPs with the size of 51.55 nm could accumulate and retain at the GBM tumor site. More importantly, we successfully demonstrated in both in vitro and in vivo studies that the NPs could elicit powerful anti-tumor efficacy by generating ROS to induce mitochondria-mediated apoptosis.The RBC@Hb@GOx NPs thus presented a promising therapeutic direction for GBM treatment. We envision that the strategy could also be useful for other primary and metastatic brain tumors.

Acknowledgements This project was supported by the Villum Fonden, Denmark, Project No. 13153. T. Zheng and M. Zhang would like to thank the China Scholarship Council (CSC) for its generous support. We would like to thank Dr. Yarong Zheng (DTU Physics) and Dr. Yuting Zhao (DTU Physics) for helping with the TEM images.

Open Access This article is licensed under a Creative Commons Attribution 4.0 International License, which permits use, sharing, adaptation, distribution and reproduction in any medium or format, as long as you give appropriate credit to the original author(s) and the source, provide a link to the Creative Commons licence, and indicate if changes were made. The images or other third party material in this article are included in the article's Creative Commons licence, unless indicated otherwise in a credit line to the material. If material is not included in the article's Creative Commons licence and your intended use is not permitted by statutory regulation or exceeds the permitted use, you will need to obtain permission directly from the copyright holder. To view a copy of this licence, visit http://creativecommons.org/licenses/by/4.0/.

Electronic supplementary material The online version of this article (https://doi.org/10.1007/s40820-020-00490-6) contains supplementary material, which is available to authorized users.

\section{References}

1. N.G. Rainov, C.M. Kramm, U. Banning, D. Riemann, H.J. Holzhausen et al., Immune response induced by retrovirusmediated HSV-Tk/GCV pharmacogene therapy in patients with glioblastoma multiforme. Gene Ther. 7(21), 1853-1858 (2000). https://doi.org/10.1038/sj.gt.3301311

2. K. Koc, I. Anik, B. Cabuk, S. Ceylan, Fluorescein sodiumguided surgery in glioblastoma multiforme: a prospective evaluation. Br. J. Neurosurg. 22(1), 99-103 (2008). https:// doi.org/10.1080/02688690701765524

3. P. Zhang, J. Miska, C. Lee-Chang, A. Rashidi, W.K. Panek et al., Therapeutic targeting of tumor-associated myeloid cells synergizes with radiation therapy for glioblastoma. Proc. Natl. Acad. Sci. U. S. A. 116(47), 23714-23723 (2019). https://doi. org/10.1073/pnas.1906346116 
4. P. Kadiyala, D. Li, F.M. Nunez, D. Altshuler, R. Doherty et al., High-density lipoprotein-mimicking nanodiscs for chemo-immunotherapy against glioblastoma multiforme. ACS Nano 13(2), 1365-1384 (2019). https://doi.org/10.1021/acsna no. 8 b06842

5. M. Ji, D.A. Orringer, C.W. Freudiger, S. Ramkissoon, X. Liu et al., Rapid, label-free detection of brain tumors with stimulated raman scattering microscopy. Sci. Transl. Med. 5(201), 201ra119 (2013). https://doi.org/10.1126/scitranslmed.30059 54

6. A. Eramo, L. Ricci-Vitiani, A. Zeuner, R. Pallini, F. Lotti et al., Chemotherapy resistance of glioblastoma stem cells. Cell Death Differ. 13(7), 1238-1241 (2006). https://doi. org/10.1038/sj.cdd.4401872

7. N.F. Brown, T.J. Carter, D. Ottaviani, P. Mulholland, Harnessing the immune system in glioblastoma. Br. J. Cancer 119(10), 1171-1181 (2018). https://doi.org/10.1038/s4141 6-018-0258-8

8. M. Zhao, D. van Straten, M.L.D. Broekman, V. Préat, R.M. Schiffelers, Nanocarrier-based drug combination therapy for glioblastoma. Theranostics 10(3), 1355-1372 (2020). https:// doi.org/10.7150/thno. 38147

9. M. Huo, L. Wang, Y. Chen, J. Shi, Tumor-selective catalytic nanomedicine by nanocatalyst delivery. Nat. Commun. 8(1), 357 (2017). https://doi.org/10.1038/s41467-017-00424-8

10. L. Feng, R. Xie, C. Wang, S. Gai, F. He, D. Yang, P. Yang, J. Lin, Magnetic targeting, tumor microenvironment-responsive intelligent nanocatalysts for enhanced tumor ablation. ACS Nano 12(11), 11000-11012 (2018). https://doi.org/10.1021/ acsnano.8b05042

11. M. Li, J. Xia, R. Tian, J. Wang, J. Fan et al., Near-infrared light-initiated molecular superoxide radical generator: rejuvenating photodynamic therapy against hypoxic tumors. J. Am. Chem. Soc. 140(44), 14851-14859 (2018). https://doi. org/10.1021/jacs.8b08658

12. D.W. Zheng, Q. Lei, J.Y. Zhu, J.X. Fan, C.X. Li et al., Switching apoptosis to ferroptosis: metal-organic network for highefficiency anticancer therapy. Nano Lett. 17(1), 284-291 (2017). https://doi.org/10.1021/acs.nanolett.6b04060

13. T.L. Whiteside, The tumor microenvironment and its role in promoting tumor growth. Oncogene 27(45), 5904-5912 (2008). https://doi.org/10.1038/onc.2008.271

14. A. Kumar, A. Tan, J. Wong, J.C. Spagnoli, J. Lam et al., Nanotechnology for neuroscience: promising approaches for diagnostics, therapeutics and brain activity mapping. Adv. Funct. Mater. 27(39), 1700489 (2017). https://doi.org/10.1002/ adfm.201700489

15. Z. Tang, Y. Liu, M. He, W. Bu, Chemodynamic therapy: tumour microenvironment-mediated fenton and fenton-like reactions. Angew. Chem. Int. Ed. 58(4), 946-956 (2019). https ://doi.org/10.1002/anie.201805664

16. P. Xue, R. Yang, L. Sun, Q. Li, L. Zhang, Z. Xu, Y. Kang, Indocyanine green-conjugated magnetic prussian blue nanoparticles for synchronous photothermal/photodynamic tumor therapy. Nano-Micro Lett. 10, 74 (2018). https://doi. org/10.1007/s40820-018-0227-z
17. T. Wang, H. Zhang, H. Liu, Q. Yuan, F. Ren et al., Boosting $\mathrm{H}_{2} \mathrm{O}_{2}$-guided chemodynamic therapy of cancer by enhancing reaction kinetics through versatile biomimetic fenton nanocatalysts and the second near-infrared light irradiation. Adv. Funct. Mater. 30(3), 1906128 (2019). https://doi.org/10.1002/ adfm.201906128

18. B. Song, Y.L. Zhang, J. Liu, X.L. Feng, T. Zhou, L.Q. Shao, Is neurotoxicity of metallic nanoparticles the cascades of oxidative stress? Nanoscale Res. Lett. 11(1), 291 (2016). https://doi. org/10.1186/s11671-016-1508-4

19. D. Duan, H. Liu, M. Xu, M. Chen, Y. Han, Y. Shi, Z. Liu, Size-controlled synthesis of drug-loaded zeolitic imidazolate framework in aqueous solution and size effect on their cancer theranostics in vivo. ACS Appl. Mater. Interfaces. 10(49), 42165-42174 (2018). https://doi.org/10.1021/acsami.8b17660

20. X. Wu, H. Yue, Y. Zhang, X. Gao, X. Li et al., Packaging and delivering enzymes by amorphous metal-organic frameworks. Nat. Commun. 10(1), 5165 (2019). https://doi.org/10.1038/ s41467-019-13153-x

21. C.Y. Sun, Y. Liu, J.Z. Du, Z.T. Cao, C.F. Xu, J. Wang, Facile generation of tumor-PH-labile linkage-bridged block copolymers for chemotherapeutic delivery. Angew. Chem. Int. Ed. 55(3), 1010-1014 (2016). https://doi.org/10.1002/anie.20150 9507

22. D. Niu, Y. Li, J. Shi, Silica/organosilica cross-linked block copolymer micelles: a versatile theranostic platform. Chem. Soc. Rev. 46(3), 569-585 (2017). https://doi.org/10.1039/ c6cs00495d

23. C. Maiti, S. Parida, S. Kayal, S. Maiti, M. Mandal, D. Dhara, Redox-responsive core-cross-linked block copolymer micelles for overcoming multidrug resistance in cancer cells. ACS Appl. Mater. Interfaces. 10(6), 5318-5330 (2018). https://doi. org/10.1021/acsami.7b18245

24. D. Shen, J. Yang, X. Li, L. Zhou, R. Zhang et al., Biphase stratification approach to three-dimensional dendritic biodegradable mesoporous silica nanospheres. Nano Lett. 14(2), 923-932 (2014). https://doi.org/10.1021/nl404316v

25. T.L. Nguyen, Y. Choi, J. Kim, Mesoporous silica as a versatile platform for cancer immunotherapy. Adv. Mater. 31(4), 1803953 (2019). https://doi.org/10.1002/adma.201803953

26. S.I. Ahn, Y.J. Sei, H.J. Park, J. Kim, Y. Ryu et al., Microengineered human blood-Brain barrier platform for understanding nanoparticle transport mechanisms. Nat. Commun. 11(1), 175 (2020). https://doi.org/10.1038/s41467-019-13896-7

27. C.B. Liu, J.Q. Chen, Y. Zhu, X.J. Gong, R.Q. Zheng et al., Highly sensitive $\mathrm{MoS}_{2}$-indocyanine green hybrid for photoacoustic imaging of orthotopic brain glioma at deep site. Nano-Micro Lett. 10, 48 (2018). https://doi.org/10.1007/s4082 0-018-0202-8

28. T. Miao, X. Ju, Q. Zhu, Y. Wang, Q. Guo, T. Sun, C. Lu, L. Han, Nanoparticles surmounting blood-brain tumor barrier through both transcellular and paracellular pathways to target brain metastases. Adv. Funct. Mater. 29(27), 1900259 (2019). https://doi.org/10.1002/adfm.201900259

29. C. Fillebeen, L. Descamps, M.P. Dehouck, L. Fenart, M. Benaïssa, G. Spik, R. Cecchelli, A. Pierce, A 
receptor-mediated transcytosis of lactoferrin through the blood-brain barrier. J. Biol. Chem. 274(11), 7011-7017 (1999). https://doi.org/10.1074/jbc.274.11.7011

30. X. Liu, Z. Shen, J. Jiang, H. Meng, Transcytosis-an effective targeting strategy that is complementary to "EPR effect" for pancreatic cancer nano drug delivery. Theranostics 9(26), 8018-8025 (2019). https://doi.org/10.7150/thno.38587

31. J. Trojan, T. R. Johnson, S. D. Rudin, J. Ilan, Treatment and prevention of rat glioblastoma by immunogenic C6 cells expressing antisense insulin-like growth factor I RNA. Science 259, 94-97 (1993). https://doi.org/10.1126/science.8418502

32. F. Ahmad, Q. Sun, D. Patel, J.M. Stommel, Cholesterol metabolism: a potential therapeutic target in glioblastoma. Cancers 11(2), 146 (2019). https://doi.org/10.3390/cancers 11020146

33. J. Feng, S. Lepetre-Mouelhi, A. Gautier, S. Mura, C. Cailleau et al., A new painkiller nanomedicine to bypass the blood-brain barrier and the use of morphine. Sci. Adv. 5(2), eaau5148 (2019). https://doi.org/10.1126/sciadv.aau5148

34. V.V. Bamm, M.E.L. Henein, S.L.J. Sproul, D.K. Lanthier, G. Harauz, Potential role of ferric hemoglobin in MS pathogenesis: effects of oxidative stress and extracellular methemoglobin or Its degradation products on myelin components. Free Radic. Biol. Med. 112, 494-503 (2017). https://doi.org/10.1016/j. freeradbiomed.2017.08.022

35. H. Fini, K. Kerman, Revisiting the nitrite reductase activity of hemoglobin with differential pulse voltammetry. Anal. Chim. Acta 1104, 38-46 (2020). https://doi.org/10.1016/j. aca.2019.12.071

36. F. Ryan, J.G. Zarruk, L. Lößlein, S. David, Ceruloplasmin plays a neuroprotective role in cerebral ischemia. Front. Neurosci. 13, 1-15 (2019). https://doi.org/10.3389/fnins .2018 .00988

37. S. Yu, Z. Chen, X. Zeng, X. Chen, Z. Gu, Advances in nanomedicine for cancer starvation therapy. Theranostics $9(26)$, 8026-8047 (2019). https://doi.org/10.7150/thno.38261

38. Z. Ren, S. Sun, R. Sun, G. Cui, L. Hong et al., A metal-polyphenol-coordinated nanomedicine for synergistic cascade cancer chemotherapy and chemodynamic therapy. Adv. Mater. 32(6), 1906024 (2019). https://doi.org/10.1002/adma.20190 6024

39. C.M.J. Hu, L. Zhang, S. Aryal, C. Cheung, R.H. Fang, L. Zhang, Erythrocyte membrane-camouflaged polymeric nanoparticles as a biomimetic delivery platform. Proc. Natl. Acad. Sci. U.S.A. 108(27), 10980-10985 (2011). https://doi. org/10.1073/pnas.1106634108

40. B.T. Luk, C.M. Jack Hu, R.H. Fang, D. Dehaini, C. Carpenter, W. Gao, L. Zhang, Interfacial interactions between natural RBC membranes and synthetic polymeric nanoparticles. Nanoscale 6(5), 2730-2737 (2014). https://doi.org/10.1039/ c3nr06371b

41. M.T. Ngo, B.A.C. Harley, Perivascular signals alter global gene expression profile of glioblastoma and response to temozolomide in a gelatin hydrogel. Biomaterials 198, 122-134 (2019). https://doi.org/10.1016/j.biomaterials.2018.06.013

42. T. Lin, P. Zhao, Y. Jiang, Y. Tang, H. Jin et al., Blood-brainbarrier-penetrating albumin nanoparticles for biomimetic drug delivery via albumin-binding protein pathways for antiglioma therapy. ACS Nano 10(11), 9999-10012 (2016). https://doi. org/10.1021/acsnano.6b04268

43. M.S. Mathew, K. Vinod, P.S. Jayaram, R.S. Jayasree, K. Joseph, Improved bioavailability of curcumin in gliadin-protected gold quantum cluster for targeted delivery. ACS Omega 4(10), 1416914178 (2019). https://doi.org/10.1021/acsomega.9b00917

44. S. Liu, Z. Dai, H. Chen, H. Ju, Immobilization of hemoglobin on zirconium dioxide nanoparticles for preparation of a novel hydrogen peroxide. Biosens. Bioelectron. 19(9), 963-969 (2004). https://doi.org/10.1016/j.bios.2003.08.025

45. J.M. Rifkind, J.G. Mohanty, E. Nagababu, The Pathophysiology of extracellular hemoglobin associated with enhanced oxidative reactions. Front. Physiol. 6, 1-7 (2015). https://doi. org/10.3389/fphys.2014.00500

46. S.M.H. Sadrzadeh, E. Graf, S.S. Panter, P.E. Hallaway, J.W. Eaton, Hemoglobin: a biologic fenton reagent. J. Biol. Chem. 259(23), 14354-14356 (1984)

47. T. Zheng, T. Zhou, X. Feng, J. Shen, M. Zhang, Y. Sun, Enhanced plasmon-induced resonance energy transfer (PIRET)-mediated photothermal and photodynamic therapy guided by photoacoustic and magnetic resonance imaging. ACS Appl. Mater. Interfaces. 11(35), 31615-31626 (2019). https://doi.org/10.1021/acsami.9b09296

48. P. Zhao, Z. Tang, X. Chen, Z. He, X. He et al., Ferrouscysteine-phosphotungstate nanoagent with neutral $\mathrm{pH}$ fenton reaction activity for enhanced cancer chemodynamic therapy. Mater. Horizons 6, 369-374 (2019). https://doi.org/10.1039/ C8MH01176A

49. D. Wang, H. Wu, S.Z.F. Phua, G. Yang, W. Qi Lim et al., Selfassembled single-atom nanozyme for enhanced photodynamic therapy treatment of tumor. Nat. Commun. 11(1), 357 (2020). https://doi.org/10.1038/s41467-019-14199-7

50. Q. Wu, Z. He, X. Wang, Q. Zhang, Q. Wei et al., Cascade enzymes within self-assembled hybrid nanogel mimicked neutrophil lysosomes for singlet oxygen elevated cancer therapy. Nat. Commun. 10(1), 240 (2019). https://doi.org/10.1038/ s41467-018-08234-2

51. T. Zheng, W. Wang, F. Wu, M. Zhang, J. Shen, Y. Sun, Zwitterionic polymer-gated $\mathrm{Au} @ \mathrm{TiO}_{2}$ core-shell nanoparticles for imaging-guided combined cancer therapy. Theranostics 9(17), 5035-5048 (2019). https://doi.org/10.7150/thno.35418

52. N.S. Goud, S.M. Ghouse, J. Vishnu, D. Komal, V. Talla et al., Synthesis of 1-benzyl-1H-benzimidazoles as galectin-1 mediated anticancer agents. Bioorg. Chem. 89, 103016 (2019). https://doi.org/10.1016/j.bioorg.2019.103016

53. W. Zhang, X. Hu, Q. Shen, D. Xing, Mitochondria-specific drug release and reactive oxygen species burst induced by polyprodrug nanoreactors can enhance chemotherapy. Nat. Commun. 10(1), 1704 (2019). https://doi.org/10.1038/s4146 7-019-09566-3

54. Q. Chen, L. Liu, Y. Lu, X. Chen, Y. Zhang et al., Tumor microenvironment-triggered aggregated magnetic nanoparticles for reinforced image-guided immunogenic chemotherapy. Adv. Sci. 6(6), 1802134 (2019). https://doi.org/10.1002/advs.20180 2134 